\title{
Deep Points Consolidation
}

\author{
Shihao $\mathrm{Wu}^{1} \quad$ Hui Huang ${ }^{2 *} \quad$ Minglun Gong $^{3} \quad$ Matthias Zwicker $^{1} \quad$ Daniel Cohen-Or ${ }^{4}$ \\ ${ }^{1}$ University of Bern $\quad{ }^{2}$ Shenzhen VisuCA Key Lab / SIAT $\quad{ }^{3}$ Memorial University of Newfoundland $\quad{ }^{4}$ Tel-Aviv University
}

\begin{abstract}
In this paper, we present a consolidation method that is based on a new representation of $3 \mathrm{D}$ point sets. The key idea is to augment each surface point into a deep point by associating it with an inner point that resides on the meso-skeleton, which consists of a mixture of skeletal curves and sheets. The deep points representation is a result of a joint optimization applied to both ends of the deep points. The optimization objective is to fairly distribute the end points across the surface and the meso-skeleton, such that the deep point orientations agree with the surface normals. The optimization converges where the inner points form a coherent meso-skeleton, and the surface points are consolidated with the missing regions completed. The strength of this new representation stems from the fact that it is comprised of both local and non-local geometric information. We demonstrate the advantages of the deep points consolidation technique by employing it to consolidate and complete noisy point-sampled geometry with large missing parts.
\end{abstract}

CR Categories: I.3.5 [Computer Graphics]: Computational Geometry and Object Modeling-Curve, surface, solid, and object representations

Keywords: Points representation, meso-skeleton, consolidation

\section{Introduction}

Objects in computer graphics are commonly represented only by their surface. However, objects are typically volumetric and their analysis and processing should consider also their volume. To account for the volume, skeletal shape representations have been widely used for shape modeling, analysis and editing. Skeletal representations usually keep their linkage to the surface. One of the best known examples is the medial axis transform (MAT), which is the set of centers of tri-tangent spheres. Each point on the surface is then represented by a point and a radius on the MAT's skeleton.

In this paper, we introduce a new representation for point sets that, similarly to the MAT, makes a link between the surface and its local volume. Each surface point is associated with an inner point that resides on a meso-skeleton [Tagliasacchi et al. 2012], which consists of skeletal curves in cylindrical regions and skeletal sheets (i.e., medial axes) elsewhere. The augmented representation is a set of line sections, each with one end on the surface and the other on the meso-skeleton. We term these augmented points, deep points, or dpoints for short. See Figure 1 for an illustration of the deep

\footnotetext{
*Corresponding author: Hui Huang (hhzhiyan@gmail.com)
}
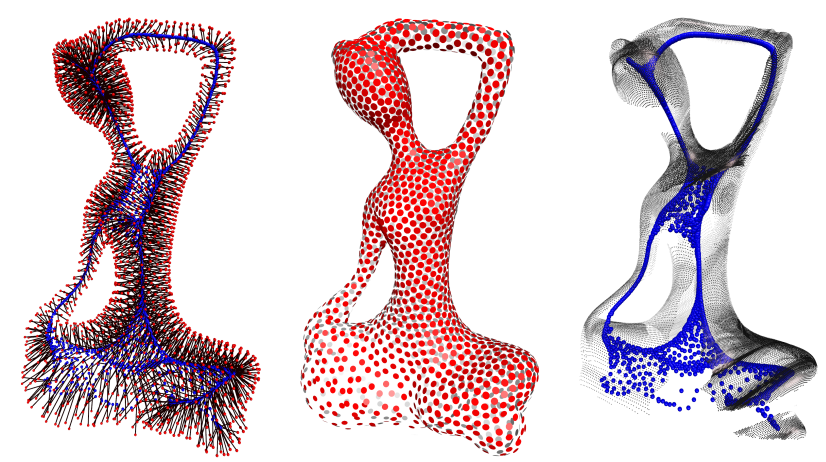

Figure 1: The deep points representation (left) is a set of line sections, each with one end (red) on the surface (middle) and the other (blue) on the meso-skeleton (right).

points representation. The deep points form a smooth mapping between the surface and meso-skeleton, where their orientations agree with their corresponding surface normal directions. It is worth noting that unlike the MAT, dpoints can be computed robustly from noisy and highly incomplete input.

The deep points representation is a result of an optimization applied to the raw input point set. The key idea is to jointly optimize the surface and skeletal points so they form a valid deep points set. As we will show, the optimization converges where the inner points form a meso-skeleton, and the surface points are consolidated. The strength of the dpoints representation stems from the fact that it is comprised of both local and non-local geometric information. We demonstrate the advantages of dpoints by employing them to consolidate and complete noisy point clouds with large missing parts.

Surface normal vectors have a critical role in surface reconstruction. Advanced consolidation techniques [Guennebaud and Gross 2007; Öztireli et al. 2009; Zheng et al. 2010; Huang et al. 2013a; Calderon and Boubekeur 2014] that can deal well with artifacts such as noise, outliers, irregular sampling, and sharp features rely on the availability of accurate normals. However, vector normals as a second order differential feature remain noisy, especially near open boundaries. Thus, unreliable normals make it challenging to complete missing surface data in the proximity of boundary points. Our consolidation technique can complete the surface without assuming the availability of accurate surface normals.

Figure 2 illustrates the main benefits of surface reconstruction using our dpoints representation. The raw scan in Figure 2(a) is nonuniform, many regions are sparse, and large parts are completely missing. Consequently, boundaries are not clearly defined and normal data is unreliable. Our dpoints representation computes a topologically correct meso-skeleton for the input shape, which provides non-local geometric information and guides the completion of missing regions on the surface. The result of our consolidated point set surface is shown in Figure 2(e), to which we apply Poisson reconstruction in Figure 2(f); see also the accompanying video.

In contrast, directly applying state-of-the-art reconstruction methods to the noisy and incomplete input, such as Poisson reconstruction [Kazhdan and Hoppe 2013] in Figure 2(b), does not provide 


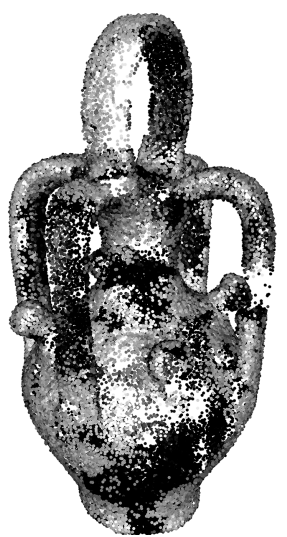

(a) Input.

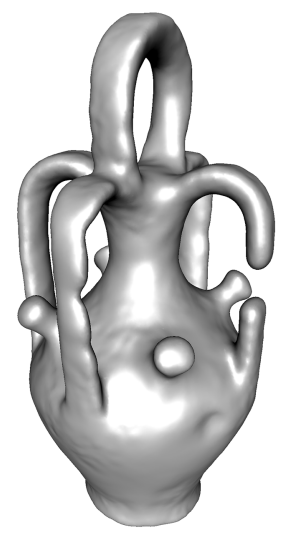

(b) Poisson recon. of (a).

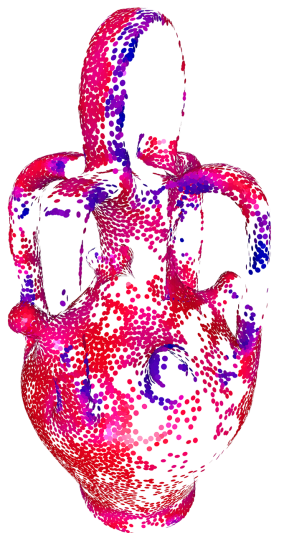

(c) WLOP consolidation.

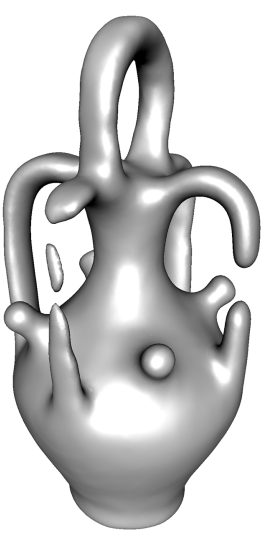

(d) Poisson recon. of (c).

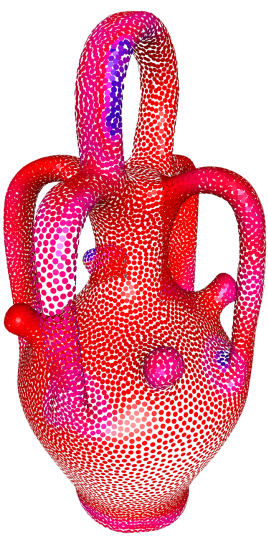

(e) Our consolidation.

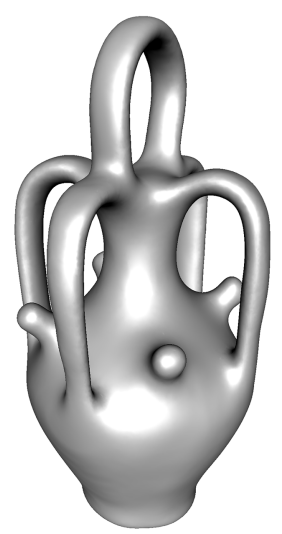

(f) Poisson recon. of (e).

Figure 2: The input point cloud (a) contains noise and large missing regions. Applying Poisson surface reconstruction [Kazhdan and Hoppe 2013] on either the input (a) or the WLOP consolidation [Huang et al. 2009] result (c) does not yield satisfactory models; see (b) and (d), respectively. The surface points shown in (e) are consolidated and completed by our dpoints technique. This leads to a much better Poisson surface reconstruction ( $f$ ). In (c) and (e), the errors of the surface point normals estimated by local PCA are evaluated based on the ground truth and color coded (blue means higher error).

plausible results. The WLOP technique [Lipman et al. 2007; Huang et al. 2009] excels in that it can consolidate raw and imperfect data without relying on normals. However, WLOP does not complete missing regions as is obvious in Figures 2(c-d).

Note that surface completion is, by its nature, an ill-posed problem. We therefore guide it by a coherent meso-skeleton, resulting in natural-looking reconstructions even for highly incomplete scans; see an evaluation in Figure 11. Rather than completing the surface with context-oblivious data, like circular [Tagliasacchi et al. 2009] or elliptical [Huang et al. 2013b] cross sections, our completion exploits the context provided by the existing data, as represented by the constructed meso-skeleton; see comparisons in Figure 12.

\section{Related work}

Surface reconstruction. In a broader context, our work is related to the vast literature on surface reconstruction [Hoppe et al. 1992; Turk and Levoy 1994; Amenta et al. 1998]. Noise is a major challenge in handling real scanned data. Assuming local smoothness, methods based on signed distance functions (SDF) [Carr et al. 2001; Kazhdan et al. 2006; Kazhdan and Hoppe 2013] can reconstruct watertight surfaces. These techniques assume the whole model is scanned and when missing data is significant the reconstructed surfaces are often overly smooth and may contain topological errors. Please refer to Berger et al. [2013; 2014] for a more comprehensive survey of state-of-the-art methods.

Surface Consolidation. Consolidation is an important preprocessing step, such as normal estimation, denoising, smoothing and regularization, which works directly on a point set itself. Early work by Alexa et al. [2001] defines a point set surface through moving least squares (MLS) projection. Later works focus on remitting the over-smoothing problem. Representative approaches include anisotropic smoothing [Lange and Polthier 2005], point-sampled cell complexes [Adamson and Alexa 2006], algebraic point set surfaces [Guennebaud and Gross 2007], robust implicit MLS [Öztireli et al. 2009] and point set resampling [Huang et al. 2013a]. To preserve sharp features, Avron et al. [2010] use a $\ell_{1}$-sparse method to compute piecewise smooth surfaces, whereas Huang et al. [2013a] generate piecewise smooth point set surfaces through point projec- tion. Recent work by Calderon and Boubekeur [2014] preserves sharp features under a point morphology framework. All of these techniques depend on oriented normals for the projection control.

The most related work to our approach is Weighted Locally Optimal Projection (WLOP) [Lipman et al. 2007; Huang et al. 2009], which generates a uniform distributed point set with oriented normals. Preiner et al. [2014] develop an accelerated version of WLOP by using a more compact representation of the original input points. WLOP-based consolidation methods are robust since they do not rely on the normals of the input points. However, none of them was designed to complete point clouds. In that sense, our method fuses consolidation and completion into one coherent technique.

Completion. Missing data, caused by self-occlusion, light absorption, or challenging surface materials [Wu et al. 2014], is one of the most challenging problems in surface reconstruction. Diffusionbased methods [Davis et al. 2002] are able to fill small holes with complex boundaries. To fill large holes, context-based [Sharf et al. 2004; Harary et al. 2014], and repetition-based [Zheng et al. 2010] methods are proposed, with the assumption that the missing parts can be replaced with other parts of the input itself. Surface evolution methods [Cohen and Cohen 1991; Esteve et al. 2005; Tagliasacchi et al. 2011], on the other hand, fill large missing data by shrinking a reasonably reconstructed initial mesh. Other methods infer missing data through exploiting high-level knowledge and priors, such as symmetry relationships [Pauly et al. 2008], volumetric smoothness [Tagliasacchi et al. 2011], canonical regularities [Li et al. 2011], and global parity measurement [Seversky and Yin 2012]. Nevertheless, these techniques cannot avoid erroneous topological reconstructions. Hence, interactive methods were developed to allow guiding the reconstruction with topology control [Sharf et al. 2007b; Yin et al. 2014].

Skeletonization. Skeletonization has been intensely studied in computer graphics, e.g., [Au et al. 2008; Cao et al. 2010; Bucksch et al. 2010; Natali et al. 2011]. In particular, curve skeleton techniques were developed in the context of surface reconstruction [Sharf et al. 2007a; Tagliasacchi et al. 2009; Li et al. 2010; Livny et al. 2010; Huang et al. 2013b]. Tagliasacchi et al. [2009] introduce a curve skeleton extraction mechanism by the use of the 


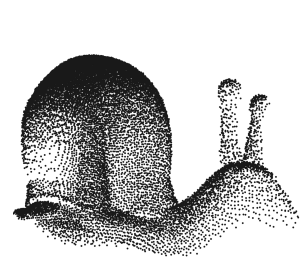

(a) Input $C$

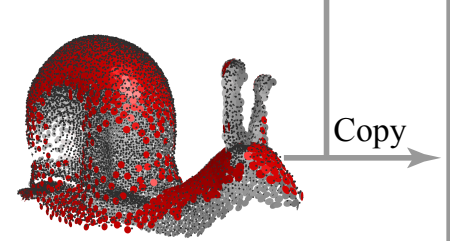

(b) Initial consolidation $S$

Joint optimization

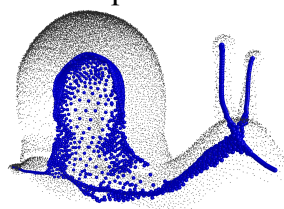

(c) Forming the meso-skeleton $Q$

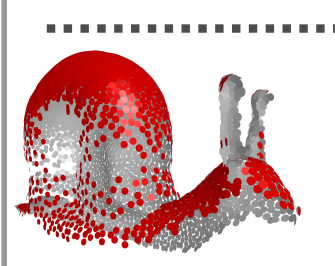

(d) Consolidating surface points $P$

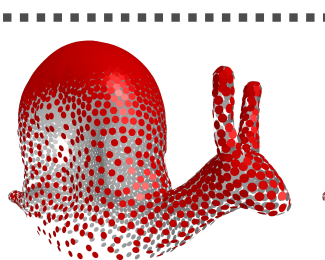

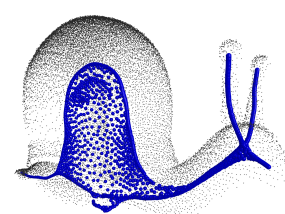

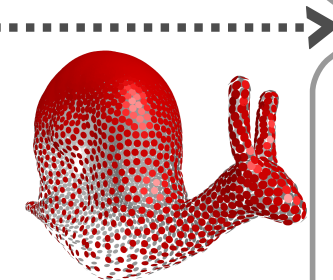

\section{$P$}

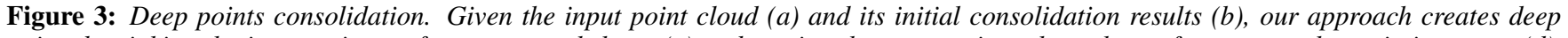
points by sinking the inner points to form a meso-skeleton (c) and moving the outer points along the surface to complete missing areas (d). The final representation consists of a set of coherent vectors that connects the surface with the meso-skeleton.

rotation symmetry axis. Huang et al. [2013b] compute $L_{1}$-medial skeletons with conditional regularization.

The work of Tagliasacchi et al. [2012] computes meso-skeletons, which combine medial sheets and curve skeletons. We adapt their notion of meso-skeletons. However, our method differs in that we do not assume having a complete surface with reliable normals. We generate the meso-skeleton as a by-product of the point cloud consolidation from noisy and incomplete input that lacks reliable normals. Miklos et al. [2010] introduce a generalization of the MAT that provides a medial representation at different levels of abstraction. While this approach is more robust to noise, it still does not address the issue of incomplete data.

To complete the missing parts along the recovered curve skeleton, these methods [Tagliasacchi et al. 2009; Huang et al. 2013b] typically run cross-sectional curve interpolation and assume that the geometry of the cross-sectional curve is circular or elliptical. Our approach for computing meso-skeletons is inspired by the skeletonization scheme of Huang et al. [2013b]. However, we introduce new adaptive and anisotropic neighborhoods (Figure 5), which allow us to generate skeletons of different topologies (2D sheets \& 1D curves) under the same framework.

\section{Deep Points}

A deep point consists of a pair of points $\left\langle p_{i}, q_{i}\right\rangle$, an outer point $p_{i} \in \mathbb{R}^{3}$ that resides on the surface and a corresponding inner point $q_{i} \in \mathbb{R}^{3}$ that resides on a meso-skeleton. We refer to the outer points $P=\left\{p_{i}\right\}_{i \in I}$ as surface points and the inner ones $Q=$ $\left\{q_{i}\right\}_{i \in I}$ as skeletal points, where the index set $I$ is the same. We represent points in space and their orientations as column vectors, and denote orientation vectors with bold fonts.

The deep points representation $\langle P, Q\rangle=\left\{\left\langle p_{i}, q_{i}\right\rangle\right\}_{i \in I} \subset \mathbb{R}^{6}$ shall satisfy the following criteria:

- the surface points $P$ reside on the latent surface and are regularly distributed;

- the collection of the skeletal points $Q$ forms a meso-skeleton of the surface, which may consists of both 2D surface sheets and $1 \mathrm{D}$ curves;
- the dpoint orientation $\mathbf{m}_{i}=\left(p_{i}-q_{i}\right) /\left\|p_{i}-q_{i}\right\|$ agrees with the surface normal $\mathbf{n}_{i}$ at the corresponding surface point $p_{i}$.

These objectives yield a joint optimization of the surface and the skeletal points under both position and orientation constraints. As the optimization converges, the deep points are generated.

We provide an overview of our algorithm in Figure 3. Let us denote the input point cloud with $C=\left\{c_{j}\right\}_{j \in J} \subset \mathbb{R}^{3}$ (Figure 3(a)). The point cloud can be unoriented and unevenly distributed, and it may have large missing parts. We start by applying WLOP consolidation [Huang et al. 2009] on $C$ to yield a denoised, oriented, and downsampled point set $S=\left\{s_{i}, \mathbf{n}_{i}\right\}_{i \in I}$ (Figure 3(b)). Through replicating the consolidated input $S$ and sinking these replicated points down into the shape interior (Section 4.1), an initial meso-skeleton (Figure 3(c), left) is formed. We refer to points on this initial meso-skeleton as anchor points and denote them by $H=\left\{h_{i}\right\}_{i \in I}$.

With set $S$ serving as the initial surface points (Figure 3(d), left) and set $H$ serving as the initial skeletal points, we optimize the set $\langle S, H\rangle$ (Figure 3(d) and (c), left to right) to form a valid deep points representation that satisfies the three criteria introduced above. In Section 4.2, we describe the optimization of the skeletal points into a connected and topologically correct meso-skeleton of the surface. The corresponding surface point consolidation is presented in Section 4.3. We show a sequence of optimized point clouds in Figure 3(c) and (d), including both skeletal and surface point sets, to demonstrate how this joint optimization converges to a valid state of deep points $\langle P, Q\rangle$ as presented in Figure 3(e).

\section{Joint Optimization on Dpoints}

The joint optimization of dpoints consists of three stages: sinking the consolidated points $S$ (Figure 3(b)) to obtain a set of anchor points $H$ (Figure 3(c), left), and then consolidating these anchor points $H$ to form a meso-skeleton (Figure 3(c), left to right), and finally consolidating a second copy of the surface points $S$ to complete the missing areas and refine the connection to the mesoskeleton (Figure 3(d), left to right). At the end, a good dpoints representation is obtained, which consists of a meso-skeleton with proper topology, a complete and consolidated point set surface, and an one-to-one mapping between the two, where the mapping 
(dpoint) orientation agrees with the surface normal (Figure 3(e)).

\subsection{Sinking Consolidated Points}

Our algorithm to obtain an initial meso-skeleton is loosely inspired by the grassfire transform for binary images. Intuitively, we set the consolidated points "on fire", causing the fire to burn from the surface into the volume of the shape. When opposing fire fronts meet, they stop propagating and form the initial meso-skeleton. We consider points that have met an opposing front as "anchored". A key challenge in this step is that we work with point sets that may have large missing parts, i.e., are not closed, which may lead to regions that never meet an opposing front. We handle this problem by exploiting the dpoints representation, that is, the connection between moving inner points and their corresponding surface points.

We initialize outer and inner points of each dpoint $\left\langle p_{i}, q_{i}\right\rangle$ by placing both at the corresponding consolidated point $s_{i}$. This gives us dpoints of zero length, where the length means the distance between the outer and inner point. To move inner points into the volume of the shape and anchor them at proper locations, we apply an iterative procedure. For each dpoint, we compute a static neighborhood $\mathcal{P}_{i}$ on the surface points, and a dynamic neighborhood $\mathcal{Q}_{i}$ on the inner points, which we update at each iteration. We use the static neighborhood to determine the amount of movement into the volume for each inner point, and to maintain smoothness among neighboring inner points. On the other hand, we use the dynamic neighborhoods to determine whether opposing fronts meet and inner points settle.

In a preprocessing step, we first compute the average sparsity $r$ of the consolidated point set $S$. That is, $r$ is the average distance to the closest neighbor,

$$
r=\frac{1}{|S|} \sum_{i \in I} \min _{i^{\prime} \in I \backslash\{i\}}\left\|s_{i}-s_{i^{\prime}}\right\|,
$$

where operator $\|\cdot\|$ computes the Euclidean norm of a vector and $|\cdot|$ measures the size of a set. We compute the static neighborhood $\mathcal{P}_{i}=\left\{p_{i^{\prime}} \mid\left\|p_{i^{\prime}}-p_{i}\right\|<\sigma_{p} r\right\}$ for each surface point $p_{i}$ where the parameter $\sigma_{p}$ defaults to 5 , and denote its set index (the set of indices belonging to $\mathcal{P}_{i}$ ) by $I_{i}^{\mathcal{P}}$.

Within each iteration, we compute the dynamic neighborhood $\mathcal{Q}_{i}=\left\{q_{i^{\prime}} \mid\left\|q_{i^{\prime}}-q_{i}\right\|<\sigma_{q} r\right\}$ with the parameter $\sigma_{q}=2$ by default, and denote a set index $I_{i}^{\mathcal{Q}}$ for each inner point $q_{i}$. We use this neighborhood to detect whether an inner point $q_{i}$ should settle. We say opposing fronts meet within the dynamic neighborhood if

$$
\max _{i^{\prime} \in I_{i}^{\mathcal{Q}}} \mathbf{n}_{i^{\prime}} \cdot \mathbf{n}_{i} \leq \cos (\omega)
$$

where $\mathbf{n}_{i}$ are the normals of surface points, which we obtained in the initial consolidation step [Huang et al. 2009]. Intuitively, the above condition means that an inner point stops sinking once it meets other inner points with sufficiently different normals. The criteria for being sufficiently different is based on the parameter $\omega$. It controls how deep the inner points sink and it defaults to $45^{\circ}$.

Next, we move each unsettled inner point by a small distance along the direction opposite to its normal, i.e., $q_{i}=q_{i}-t \mathbf{n}_{i}$. In the first iteration, the moving distance $t$ is set to $r / 2$ for all unsettled inner points. In the following iterations, we adaptively compute $t$ for each inner point $q_{i}$. For each $q_{i}$, we set $t$ as the average moving distance in the previous iteration of all inner points (settled and unsettled) in its static neighborhood $\left\{q_{i^{\prime}}\right\}_{i^{\prime} \in I_{i}^{\mathcal{P}}}$.

After the inner points advance to the new locations, we further adjust their positions to maintain the smoothness among neighboring
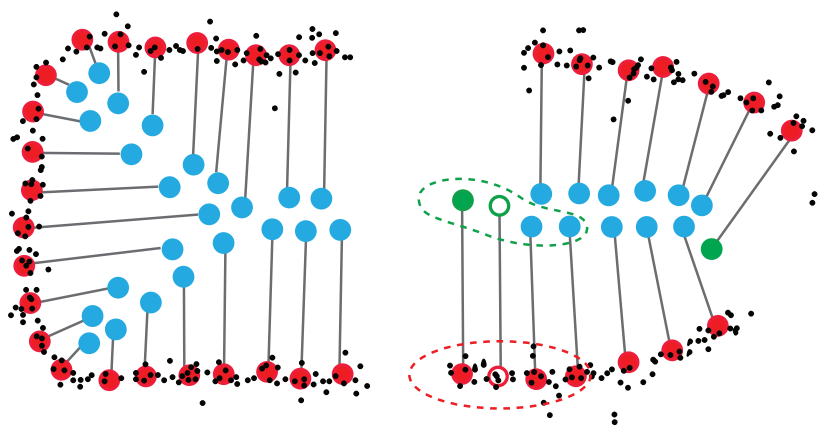

Figure 4: Generating anchor points by sinking consolidated (red) points. Most inner points (blue) stop sinking as they get close to other inner points with different normals, but some (green) need to be stopped through bilateral smoothing. The dashed green curve shows the neighborhood used for one inner point (hollow green) during smoothing. This neighborhood is static and is determined based on the corresponding surface point (hollow red).

inner points. We smooth inner point positions using a form of crossbilateral filtering, where the filter weights are determined based on the surface points $\mathcal{P}_{i}$ in the static neighborhood of each inner point. In particular, the weights measure the proximity and normal similarity of neighboring surface points. This leads to the cross-bilateral filter updates of inner point positions $q_{i}$ as:

$$
q_{i}=\frac{\sum_{i^{\prime} \in I_{i}^{\mathcal{P}}} \theta\left(p_{i}, p_{i^{\prime}}\right) \phi\left(\mathbf{n}_{i}, \mathbf{n}_{i^{\prime}}\right) q_{i^{\prime}}}{\sum_{i^{\prime} \in I_{i}^{\mathcal{P}}} \theta\left(p_{i}, p_{i^{\prime}}\right) \phi\left(\mathbf{n}_{i}, \mathbf{n}_{i^{\prime}}\right)}
$$

with the bilateral weighting functions:

$$
\theta\left(p_{1}, p_{2}\right)=e^{-\left(\frac{\left\|p_{1}-p_{2}\right\|}{r}\right)^{2}}, \phi\left(\mathbf{n}_{1}, \mathbf{n}_{2}\right)=e^{-\left(\frac{1-\mathbf{n}_{1}^{T} \mathbf{n}_{2}}{1-\cos (\omega)}\right)^{2}} .
$$

Here the parameter $r$ is computed in $\mathrm{Eq}(1)$ and the parameter $\omega$ is the same one used in Eq (2).

It is worth noting the importance of using the static neighborhood $\mathcal{P}_{i}$ over surface points, and the positions and normals of surface points in this neighborhood, to compute the weights in Eq (3). As shown in Figure 5(d), this strategy helps the inner points to maintain their structure as they move into the volume. In addition, since both settled and unsettled points are used for smoothing, an unsettled point may stop traveling and be marked as settled after most of its neighbors are settled. This feature is very important for handling highly incomplete point clouds because there may be points that will never run into other points as determined by Eq (2). They hence would never stop traveling along their anti-normal directions; see e.g., the green points in Figure 4.

\subsection{Forming the Meso-Skeleton}

Once all the inner points settle at the interior of the 3D shape, they form a set of fixed anchor points $H=\left\{h_{i}\right\}_{i \in I}$. As shown in Figure $5(\mathrm{~d})$, these anchor points do not necessarily form an intuitive meso-skeleton consisting of connected curves and surfaces. In addition, due to the missing data, there are sparse areas where the anchor points still leave gaps. We compute a point-based mesoskeleton with topologically-correct connections by further consolidating the inner points. We formulate an optimization with two objectives: first, to keep the inner points close to the $L_{1}$-median of their neighboring anchor points; second, to uniformly distribute them along connected curves and surface sheets. 


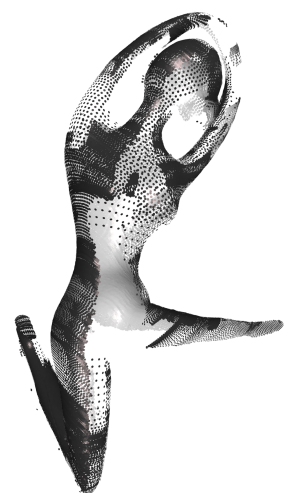

(a) Input $(C)$.

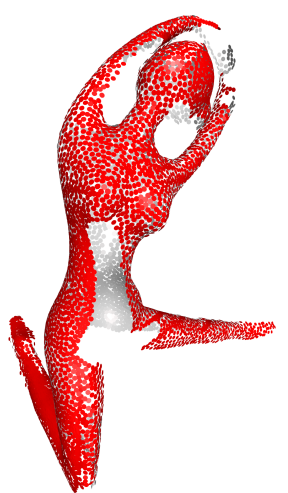

(b) $\operatorname{WLOP}(S)$.

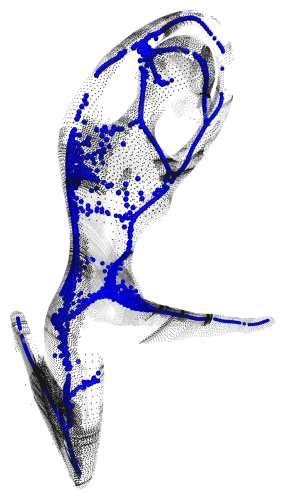

(c) Contraction of (b).

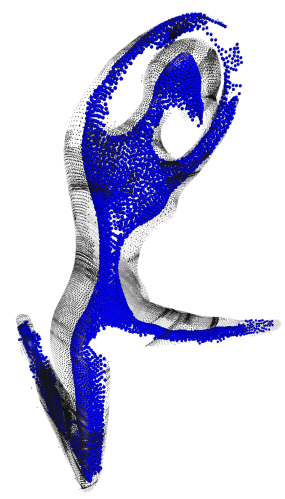

(d) Anchor points $(H)$.

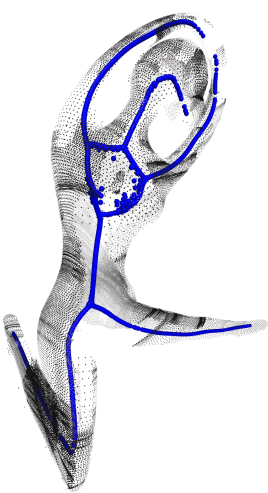

(e) Contraction of (d).

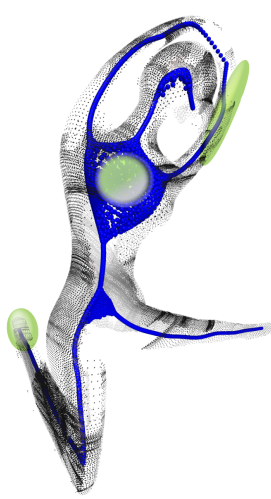

(f) Meso-skeleton.

Figure 5: Given a highly incomplete raw scan (a) and its WLOP consolidated points (b), direct point contraction fails to provide a good set of skeletal points $(c)$. Sinking the inner points first until they settle into anchor points $(d)$ and then applying contraction yields cleaner skeletal curves (e), but does not form skeletal surface sheets. Using anisotropic repulsion, our approach generates the meso-skeleton $(f)$ with mixed curves and surface sheets in a uniform framework. The green ellipsoids in $(f)$ show the anisotropic neighborhoods used in three areas.

The key issue in implementing both objectives is to determine a proper anisotropic neighborhood for each inner point. In cylindrical areas, we are aiming to obtain skeletal curves, hence neighborhoods of long and thin prolate ellipsoid shapes are appropriate. For plane-like areas, we want to generate skeletal surface sheets, and flat neighborhoods of large oblate ellipsoid shapes would work best. Finally, in areas near the endpoints of skeletal curves or at boundaries of skeletal surfaces, a small ellipsoid should be used to avoid shrinkage of the skeletal curves or surfaces.

To automatically determine the appropriate elliptical neighborhood at an inner point $q_{i}$, we first apply PCA on inner points within $q_{i}$ 's neighborhood $\mathcal{Q}_{i}$ (as computed in Section 4.1). We use the three principle axes $\left\{\mathbf{v}_{i}^{1}, \mathbf{v}_{i}^{2}, \mathbf{v}_{i}^{3}\right\}$ as the local coordinates of the ellipsoid. Instead of using the PCA eigenvalues to determine the semi-axis lengths of the ellipsoid, however, we develop a different procedure exploiting our dpoints representation. We will discuss the benefits of this below. Let us denote the semi-axis lengths by scalars $\left\{l_{i}^{1}, l_{i}^{2}, l_{i}^{3}\right\}$. We compute them by first projecting the normal directions of all inner points within the neighborhood $\mathcal{Q}_{i}$ to the corresponding principle axis and then computing the average normal projection length. The larger the projection length, the shorter the semi-axis length $l$ is set to. To be precise, we set

$$
l_{i}^{m}=\left(\frac{\sum_{i^{\prime} \in I_{i}^{\mathcal{O}}}\left|\mathbf{n}_{i^{\prime}}^{T} \mathbf{v}_{i}^{m}\right|}{\left|\mathcal{Q}_{i}\right|}+\epsilon\right)^{-1}, \quad m=1,2,3 .
$$

The constant parameter $\epsilon$ is small and set to 0.1 by default.

As shown in Figure 5(f) and illustrated in Figure 6, the ellipsoids computed can automatically adapt to the local topology of the inner points. In the center regions of the skeletal curves or skeletal surface sheets, where surface normals are perpendicular to the dominant PCA directions, the normal projection length is small, resulting in a large semi-axis length. This helps to connect gaps on skeletal curves and close holes on skeletal surface sheets. On the other hand, at the end points of skeletal curves and the borders of skeletal surface sheets, the normal projection length is large even along the dominant PCA directions. Hence, a small semi-axis length is used to avoid shrinkage in these areas, because the inner points are constrained to smaller regions.

With the anisotropic neighborhood defined, we now formulate the meso-skeleton as the result of an optimization that attempts, first, to

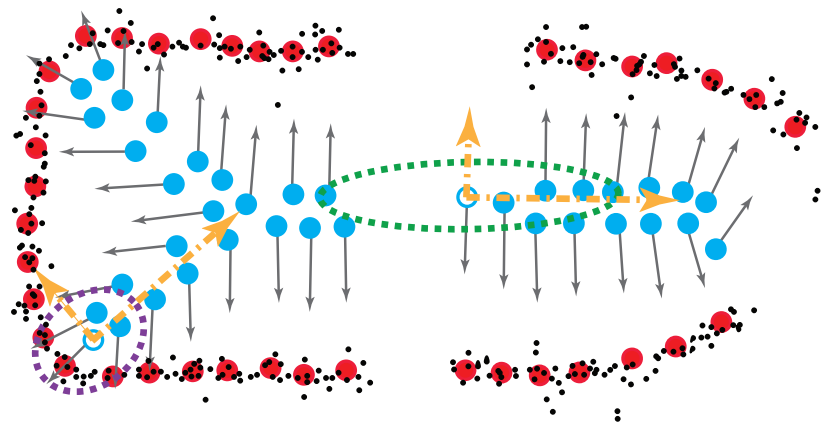

Figure 6: The anisotropic neighborhoods (dashed ovals) defined for two inner points along their local PCA axes (orange arrows). Even though the eigenvalues along the dominant PCA direction are high in both cases, the normal projection lengths are different, resulting in the green oval being much longer than the purple one.

keep the inner points close to the $L_{1}$-median of their neighboring anchor points, and second, to consolidate the inner points to form regularly sampled, connected skeletal curves and surfaces. Hence, the optimization is a sum of a data and a regularization term:

$$
\underset{Q}{\operatorname{argmin}} \sum_{i \in I} \sum_{k \in I} \vartheta\left(q_{i}, h_{k}\right)\left\|q_{i}-h_{k}\right\|+R(Q) .
$$

The first term (data term) is the weighted $L_{1}$-median, where the weight function $\vartheta\left(q_{i}, h_{k}\right)=e^{-d_{e}^{2}\left(q_{i}, h_{k}\right) / r^{2}}$ is defined based on the ellipsoid (Mahalanobis) distance between the neighboring anchor point $h_{k}$ and the center point $q_{i}$. This distance function returns the same constant number for all points on the ellipsoid surface defined at $q_{i}$; and hence gives them equal weights. It is computed as

$$
d_{e}\left(q_{i}, h_{k}\right)=\left\|\mathbf{A}_{i}^{T}\left(q_{i}-h_{k}\right)\right\|,
$$

where the $3 \times 3$ column matrix $\mathbf{A}_{i}=\left[\mathbf{v}_{i}^{1} / l_{i}^{1} ; \mathbf{v}_{i}^{2} / l_{i}^{2} ; \mathbf{v}_{i}^{3} / l_{i}^{3}\right]$ consists of the semi-axes of our elliptical neighborhoods; see green ellipsoids in Figure 5(f) as an illustration.

Applying the $L_{1}$-median term alone tends to yield a sparse but nonregular point distribution; see Figure $5(\mathrm{e})$ where local $L_{1}$-median 


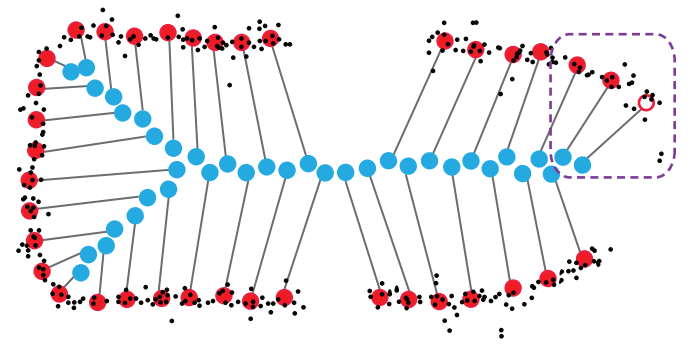

(a) meso-skeleton

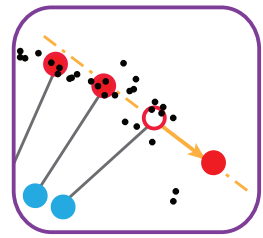

(b) Repulsion force

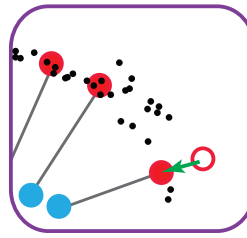

(c) Fitting with prior

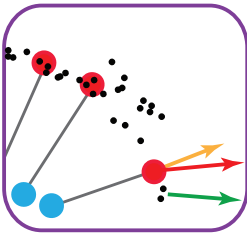

(d) normal update
Figure 7: Once the meso-skeleton (a) is formed, we consolidate the surface points through alternatively optimizing surface point locations and updating their normals. The repulsion force introduced by the regularization term pushes the point along the tangential (orange line) direction (b), whereas the data fitting term with the shape prior moves the point towards the local $L_{1}$-median with adjustment on the dpoint length (c). (d) Once the optimal location is found, the normal direction is updated based on dpoint orientation (orange arrow) and PCA normal (green arrow).

centers are accumulated in clusters. We avoid clustering using the regularization term $R(Q)$, which represents additional repulsion forces. Our approach is derived from previous ones [Lipman et al. 2007; Huang et al. 2009], but with important differences. The key idea is that to allow the inner points to form thin skeletal curves or surfaces, we cannot repulse points along the directions that are perpendicular to the skeletal curves or surfaces. Hence, we use our elliptical neighborhoods to define novel anisotropic repulsion forces. The regularization term is a sum of these forces at each inner point $q_{i}$, summed over all inner points $i \in I$ with $\left\{\lambda_{i}\right\}$,

$$
R(Q)=\sum_{i \in I} \lambda_{i} \sum_{i^{\prime} \in I \backslash\{i\}} \frac{\vartheta\left(q_{i}, q_{i^{\prime}}\right)}{\left\|d_{e}\left(q_{i}, q_{i^{\prime}}\right)\right\|^{3}} .
$$

Intuitively, the repulsion force between an inner point $q_{i} \in \mathcal{Q}$ and a neighbor $q_{i}^{\prime}, i^{\prime} \in I \backslash\{i\}$ is given by the projection of the neighbor into the local coordinates of the ellipsoid $\left\{\mathbf{v}_{i}^{1}, \mathbf{v}_{i}^{2}, \mathbf{v}_{i}^{3}\right\}$, and applying stronger repulsion along the direction where the semi-length of the ellipsoid is longer. The balancing weight $\lambda_{i}$ is determined by a regularization parameter $\mu$ that we define next in this subsection.

It is also worth noting that our anisotropic regularization is different from that of the $L_{1}$-medial skeleton [Huang et al. 2013b]. There the conditional regularization stops to push points whenever the skeletal points are forming a curve structure. Under the same situation, our anisotropic repulsion still pushes points along the curve direction, allowing them to connect broken skeletal curves and to obtain a more uniform point distribution. This not only removes the need of finding bridge points to connect broken curves, but also handles different topologies (curves and surface sheets) under a uniform framework.

When the gradient of the energy in Eq (4) equals to zero, the fol- lowing relation is satisfied at each $q_{i}$ with fixed coefficients:

$$
\begin{aligned}
& \sum_{i^{\prime} \in I \backslash\{i\}} \alpha_{i i^{\prime}}\left(q_{i}-q_{i^{\prime}}\right)-\lambda_{i} \sum_{i^{\prime} \in I \backslash\{i\}} \beta_{i i^{\prime}} \mathbf{A}_{i}^{T} \mathbf{A}_{i}\left(q_{i}-q_{i^{\prime}}\right)=0, \\
& \text { where } \alpha_{i i^{\prime}}=\frac{\vartheta\left(q_{i}, q_{i^{\prime}}\right)}{\left\|q_{i}-q_{i^{\prime}}\right\|} \text { and } \beta_{i i^{\prime}}=\frac{\vartheta\left(q_{i}, q_{i^{\prime}}\right)}{\left\|d_{e}\left(q_{i}, q_{i^{\prime}}\right)\right\|^{5}} \text {. }
\end{aligned}
$$

Applying a fixed point iteration, we update inner points by

$q_{i}=\frac{\sum_{i^{\prime} \in I \backslash\{i\}} \alpha_{i i^{\prime}} q_{i^{\prime}}}{\sum_{i^{\prime} \in I \backslash\{i\}} \alpha_{i i^{\prime}}}+\mu\left\|\ell_{i}\right\|^{2} \frac{\sum_{i^{\prime} \in I \backslash\{i\}} \beta_{i i^{\prime}} \mathbf{A}_{i}^{T} \mathbf{A}_{i}\left(q_{i}-q_{i^{\prime}}\right)}{\sum_{i^{\prime} \in I \backslash\{i\}} \beta_{i i^{\prime}}}$.

Here we have $\mu\left\|\ell_{i}\right\|^{2}=\lambda_{i} \sum_{i^{\prime} \in I \backslash\{i\}} \beta_{i i^{\prime}} / \sum_{i^{\prime} \in I \backslash\{i\}} \alpha_{i i^{\prime}}$ and the column vector $\ell_{i}=\left[l_{i}^{1} ; l_{i}^{2} ; l_{i}^{3}\right]$. We empirically set the regularization parameter $\mu=0.4$ by default to control the global level of anisotropic penalty applied on accumulated points.

\subsection{Surface Point Consolidation}

As shown in Figure 5, after the second stage, the inner points form a complete meso-skeleton (2D sheets \& $1 \mathrm{D}$ curves) with a regular point distribution. The meso-skeleton now provides the non-local information that can guide surface point consolidation to complete large missing areas. In practice, the location of each $p_{i}$ is adjusted through optimization based on the following objectives: i) $p_{i}$ resides on the underlying surface and hence shall be close to the $L_{1}$ median of nearby input points; ii) the points $\left\{p_{i}\right\}$ shall be regularly distributed on the underlying surface; and iii) in incompletely scanned areas where input points are missing, additional shape priors are needed to guide surface completion. See also Figure 7.

Based on the above objectives, we define the optimization function for surface points as the sum of three terms: a $L_{1}$-median data term, a regularization term $\hat{R}$, and a shape prior term $G$ :

$$
\underset{P}{\operatorname{argmin}} \sum_{i \in I} \eta\left(p_{i}\right) \sum_{c_{j} \in \mathcal{C}_{i}} \theta\left(p_{i}, c_{j}\right)\left\|p_{i}-c_{j}\right\|+\hat{R}(P)+G(P) .
$$

The $\theta$ function in the data term is the same as the one defined in Eq (3), and $\mathcal{C}_{i}=\left\{c_{j} \mid\left\|c_{j}-p_{i}\right\|<\sigma_{p} r\right\}$ is a subset of input points in $p_{i}$ 's neighborhood. The data term is weighted using a function $\eta\left(p_{i}\right)=1+\sum_{c_{j} \in \mathcal{C}_{i}} \theta\left(c_{j}, p_{i}\right)$, which outputs high weight when the density of the input points in $\mathcal{C}_{i}$ is high. Hence, when there are sufficient data in the neighborhood of $p_{i}$, the data term has higher weight to ensure the position of $p_{i}$ fits the data.

The second regularization term applies repulsion forces introduced from all neighboring surface points. To avoid points being pushed away from the surface, here the repulsion is performed along the local tangent plane at $p_{i}$. That is:

$$
\hat{R}(P)=\sum_{i \in I} \sum_{i^{\prime} \in I \backslash\{i\}} \frac{\theta\left(p_{i}, p_{i^{\prime}}\right)}{\left\|\mathbf{B}_{i}^{T}\left(p_{i}-p_{i^{\prime}}\right)\right\|^{3}},
$$

where the $3 \times 2$ projection column matrix $\mathbf{B}_{i}=\left[\mathbf{u}_{i}^{1} ; \mathbf{u}_{i}^{2}\right]$, with $\left\{\mathbf{u}_{i}^{1}, \mathbf{u}_{i}^{2}\right\}$ being two arbitrary orthogonal directions on $p_{i}$ 's tangential plane, ensures $\hat{R}\left(p_{i}\right)$ only introduces repulsion forces perpendicular to the surface normal $\mathbf{n}_{i}$.

When the point set surface in the neighborhood of $p_{i}$ is complete, repulsion forces from all directions maintain the regularity of the point distribution. Otherwise, if $p_{i}$ is near the open boundary of missing areas, its neighboring points will push it towards the hole, allowing small gaps on the surface being naturally filled. However, when the scan contains large missing areas, e.g., the object is only 
scanned from one side as in Figure 11(a), additional shape priors are needed to further guide the completion.

A big benefit introduced by the dpoints representation is the notion of volume, i.e., the length of a given dpoint $\left\|p_{i}-q_{i}\right\|$ indicates how far the surface is away from the local meso-skeleton at location $p_{i}$. This allows us to incorporate different types of shape priors with ease. For example, under the assumption that surface points in areas with missing data shall preserve a similar volume as their neighbors, we can define a volume preserving shape prior term as:

$$
G(P)=\frac{1}{2} \sum_{i \in I} \gamma\left(p_{i}\right)\left(\left\|p_{i}-q_{i}\right\|-L\left(p_{i}\right)\right)^{2},
$$

where $L\left(p_{i}\right)=\frac{\sum_{i^{\prime} \in I_{i}^{P}} \theta\left(p_{i}, p_{i^{\prime}}\right)\left\|p_{i^{\prime}}-q_{i^{\prime}}\right\|}{\sum_{i^{\prime} \in I_{i}^{P}} \theta\left(p_{i}, p_{i^{\prime}}\right)}$ computes the average dpoint length within $p_{i}$ 's neighborhood, and the weight function $\gamma\left(p_{i}\right)=\left(1+\operatorname{var}\left(\left\{\left\|p_{i^{\prime}}-q_{i^{\prime}}\right\|\right\}_{i^{\prime} \in I_{i}^{P}}\right)\right)^{-1}$ assigns high weight when the dpoint length variance within the neighborhood is low.

In other cases, we may want to minimize the volume of the reconstructed shape. To achieve this, we can define a minimal volume shape prior term as:

$$
G(P)=\frac{1}{2} \sum_{i \in I} \gamma\left(p_{i}\right)\left(L\left(p_{i}\right)\right)^{2} .
$$

Similarly as solving Eq (4), we can solve Eq (6) and update surface points by applying a fixed point iteration. For example, when the volume preserving shape prior from $\mathrm{Eq}(8)$ is used, the optimization can be solved by:

$$
\begin{aligned}
p_{i} & =\frac{\eta\left(p_{i}\right) \sum_{c_{j} \in \mathcal{C}_{i}} \alpha_{i j} c_{j}}{\gamma\left(p_{i}\right)+\eta\left(p_{i}\right) \sum_{c_{j} \in \mathcal{C}_{i}} \alpha_{i j}}+\frac{\gamma\left(p_{i}\right)\left(q_{i}+L\left(p_{i}\right) \mathbf{m}_{i}\right)}{\gamma\left(p_{i}\right)+\eta\left(p_{i}\right) \sum_{c_{j} \in \mathcal{C}_{i}} \alpha_{i j}} \\
& +\hat{\mu} \frac{\sum_{i^{\prime} \in I \backslash\{i\}} \hat{\beta}_{i i^{\prime}} \mathbf{B}_{i}^{T} \mathbf{B}_{i}\left(p_{i}-p_{i^{\prime}}\right)}{\sum_{i^{\prime} \in I \backslash\{i\}} \hat{\beta}_{i i^{\prime}}},
\end{aligned}
$$

with weights $\alpha_{i j}=\frac{\theta\left(p_{i}, c_{j}\right)}{\left\|p_{i}-c_{j}\right\|}$ and $\hat{\beta}_{i i^{\prime}}=\frac{\theta\left(p_{i}, p_{i^{\prime}}\right)}{\left\|\mathbf{B}_{i}^{T}\left(p_{i}-p_{i^{\prime}}\right)\right\|^{5}}$. The parameter $\hat{\mu}=\sum_{i^{\prime} \in I \backslash\{i\}} \hat{\beta}_{i i^{\prime}} /\left(\gamma\left(p_{i}\right)+\eta\left(p_{i}\right) \sum_{c_{j} \in \mathcal{C}_{i}} \alpha_{i j}\right)$ that controls the tangential repulsion forces defaults to 0.4 .

Once the location of surface point $p_{i}$ is adjusted, its corresponding surface normal $\mathbf{n}_{i}$ is updated. Using a similar idea as above, when dense input data is available in the local neighborhood, higher weight is given to the conventional oriented PCA normal $\tilde{\mathbf{n}}_{i}$; otherwise, we set $\mathbf{n}_{i}$ closer to the dpoint orientation $\mathbf{m}_{i}$ :

$$
\mathbf{n}_{i}=\frac{\left(\eta\left(p_{i}\right)-1\right) \tilde{\mathbf{n}}_{i}+\mathbf{m}_{i}}{\left\|\left(\eta\left(p_{i}\right)-1\right) \tilde{\mathbf{n}}_{i}+\mathbf{m}_{i}\right\|} .
$$

Alternatively optimizing the locations of surface points and updating their normals allows these surface points to redistribute along the underlying surface, converging to a regular distribution with missing areas completed. In addition, since the normal calculation considers the dpoint orientation $\mathbf{m}_{i}$, the two orientations generally agree with each other in the final dpoints representation.

\section{Results}

We test our deep point consolidation technique on various incomplete scans of both physical and virtual objects. The input data in Figures $1,5,9,10,12,14$ and 15 was acquired using a laser scanner, the ones in Figure 13(a) were downloaded from the SHREC 2015 dataset [NIST 2015], and those used in Figure 11 are digital scans of a synthetic model.

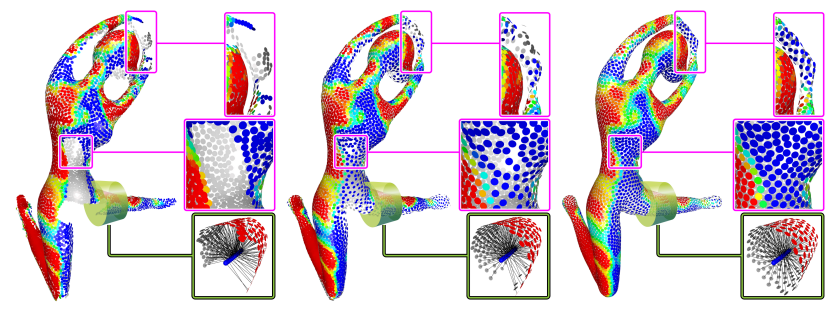

Figure 8: The initial consolidated points contain large missing areas (left). The surface consolidation process gradually closes the gaps (middle) and finally converges to a set of complete surface points (right). The color of surface points encodes the density of local input points.

Table 1: Timing for dpoints computation on all examples presented. $|C|$ and $|S|$ denote numbers of raw input points and deep points, respectively. $T_{w}$ and $T_{d}$ are the time (in sec.) needed for the initial WLOP consolidation and then dpoints consolidation, respectively.

\begin{tabular}{||l||c|c||c|c|}
\hline & $|C|$ & $|S|$ & $T_{w}$ & $T_{d}$ \\
\hline Fig. 1 & 84772 & 2143 & 3.1 & 26.3 \\
\hline Fig. 5 \& 8 & 58710 & 6870 & 4.8 & 41.2 \\
\hline Fig. 9 & 22148 & 5234 & 2.7 & 30.5 \\
\hline Fig. 10 T & 43545 & 3996 & 4.1 & 83.9 \\
\hline Fig. 10 B & 78630 & 6727 & 8.4 & 87.3 \\
\hline Fig. 12 T & 24292 & 13812 & 3.2 & 112.9 \\
\hline Fig. 12 B & 32614 & 7252 & 5.5 & 61.5 \\
\hline Fig. 13 T & 40509 & 40509 & 10.5 & 259.6 \\
\hline Fig. 13 B & 67131 & 67131 & 18.9 & 408.8 \\
\hline Fig. 14 T & 175312 & 19721 & 17.8 & 197.3 \\
\hline Fig. 14 B & 300283 & 57791 & 47.3 & 449.9 \\
\hline Fig. 15 T & 292910 & 32142 & 30.3 & 355.3 \\
\hline Fig. 15 B & 483583 & 47083 & 134.9 & 723.7 \\
\hline
\end{tabular}

Parameters and timing. On average, the initial WLOP consolidation needs 30-40 iterations to converge, whereas the iterations needed for generating deep points (consisting of sinking WLOP points into anchor points, forming meso-skeleton, and consolidating the surface) vary from 60 to 120 depending on how large the missing data regions are. Table 1 presents computation time on an Intel(R) Core(TM) i7-5930K CPU@3.5GHz with 32GB RAM.

There are two key controllable parameters in our approach: the point settling threshold $\omega$ and the neighborhood size parameter $\sigma_{p}$ used for both sinking and surface point consolidation. All other parameters $\left\{\sigma_{q}, \mu, \hat{\mu}, \epsilon\right\}$ are not sensitive to the inputs, and are fixed at the aforementioned default values for all experiments.

Figure 9 illustrates the impact of $\omega$ on the results. Generally speaking, since the inner points settle sooner under smaller $\omega$, the mesoskeleton follows the surface details more closely but is also more sensitive to noise. With larger $\omega$, the inner points often travel deeper before they settle. When they cannot meet the opposing front due to missing data, the final skeleton may deviate from the proper medial position, causing the final consolidated shape having a bigger volume. In addition, some small branches may not be represented in the meso-skeleton as well. Figure 10 illustrates that larger $\sigma_{p}$ value often leads to more uniform surface point distribution and makes the representation more robust against input noise and large missing parts. However, on the other hand, the consolidated surface may not represent the surface details as well. 


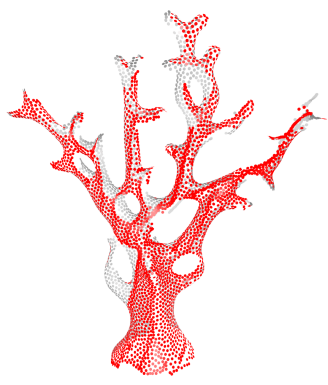

(a) WLOP.

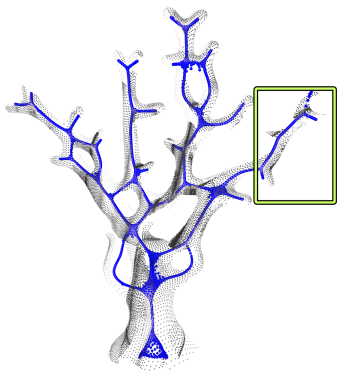

(f) Meso-skeleton from (e).

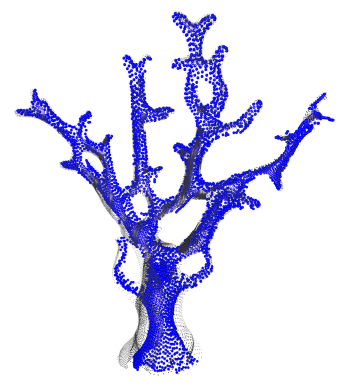

(b) Anchor points $\left(\omega=25^{\circ}\right)$

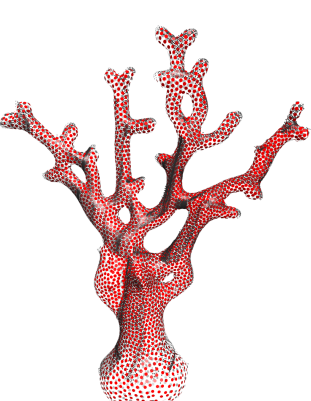

(g) Consolidation using (f)

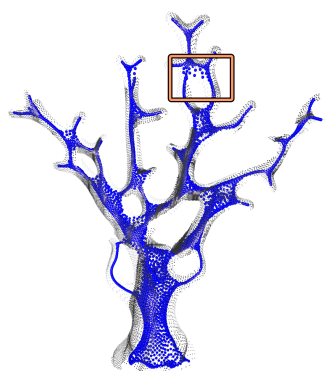

(c) Meso-skeleton from (b).

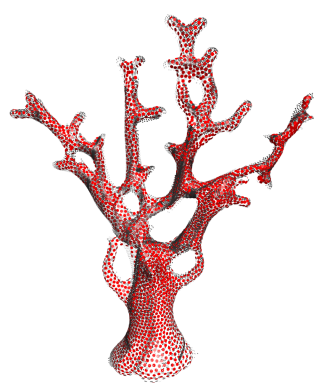

(d) Consolidation using (c).

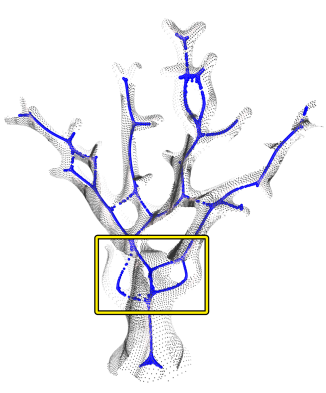

(i) Meso-skeleton from (h).

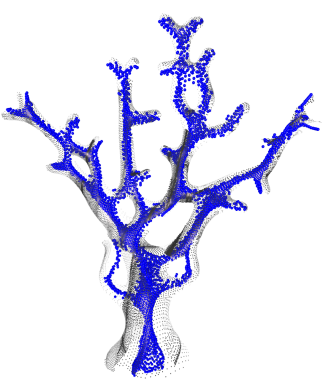

(e) Anchor points $\left(\omega=45^{\circ}\right)$.

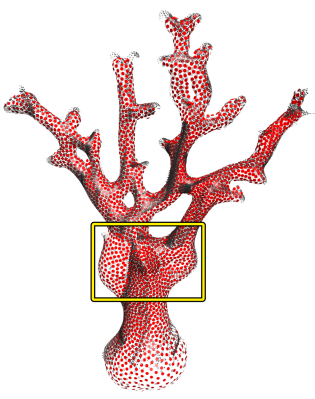

(j) Consolidation using (i).

Figure 9: The impact of the parameter $\omega$. With a small $\omega$ value $(b-d)$, the inner points settle as anchor points sooner $(b)$, the meso-skeleton $(c)$ consists of many $2 D$ sheet structures and a small number of isolated points (orange box), and the final surface point set (d) follows the input data reasonably well. In contrast, under a large $\omega$ value ( $h-j)$, the inner points have to sink deeper before they settle ( $h$ ), the meso-skeleton ( $i)$ consists of mostly $1 D$ skeletal curves and may miss fine branches (green box in $f$ ). In addition, when a large part of a cylindrical surface is missing, the location of the skeletal curve may drift away from the center (yellow box in $i$ ), causing the consolidated surface points ( $j$ ) having a much thinker cylinder and small holes on the model being improperly filled (yellow box in $j$ ).

Comparison to skeletonization and reconstruction. Figure 8 shows how the consolidation process gradually closes missing areas by adjusting the location of surface points. The benefit of having consolidated surface points is shown in Figures 2, 12, 13, and 14 , where the models reconstructed from these points are noticeably more faithful to the original shape than the ones generated directly from the input or leveraging previous skeletonization techniques [Tagliasacchi et al. 2009; Huang et al. 2013b]. In particular, through pushing surface points in an anisotropic manner, our reconstruction results better preserve the connectivity of different parts (Figures 2, 12 and 14 (bottom)), while avoiding improperly fusing them together (Figure 13). The notion of local volume of dpoints also allows the reconstruction to better preserve thin structures (Figure 14 (top)). Furthermore, Figures 11 and 12 show that our approach can infer reasonable geometries when the objects are scanned from one side only. In comparison, existing approaches such as [Tagliasacchi et al. 2011] would fail in these cases as the thickness of the objects cannot be estimated.

In terms of the meso-skeleton generated, Figure 12 shows that our inner skeleton can better respect the topology of the input shape than existing skeletonization approaches [Tagliasacchi et al. 2009; Huang et al. 2013b]. Unlike the existing approaches, which produce skeletal curves regardless surface topology, the meso-skeletons that we generated for non-tubular shapes nicely form 2D skeletal surface sheets; see, e.g., the flat surfaces in Figures 12 and 15, the cylindrical and disk shapes in Figure 14.

Reconstruction accuracy. To quantitatively evaluate the reconstruction accuracy, digital virtual scans of a synthetic model are used. With the input model serving as the ground truth, the reconstruction error is measured using the average distance between all $40 \mathrm{~K}$ vertices on the input model and their closest points on the reconstructed surface. As shown in Figure 11(a), the models reconstructed using dpoints are more favorable than the one reconstructed from input points directly. Also, as expected, the improvement is more pronounced for incomplete scans (i.e., one or two scans are used) than complete scans. Note that since the virtual scan is noise free, employing consolidation methods such as WLOP, would not improve the direct Poisson reconstruction.

Figure 11(b) further evaluates the reconstruction accuracy over noise corrupted scans. Here, Gaussian noise with $2 \%$ magnitude of the model dimension is added to the scan data. The accuracy of Poisson reconstruction over WLOP consolidation results and dpoints consolidation are plotted. The comparison again demonstrates the benefits of using our dpoints consolidation.

Sharp features and fine details. Since the dpoints representation is computed based on a small set of WLOP points downsampled from the input, it cannot fully preserve fine geometry details and sharp features on the original model; compare e.g., examples in Figures 15(b) and (d). Nevertheless, additional post-processing can be applied to reconstruct these sharp features and high-frequency details from the input. Here, the edge-aware point resampling technique (EAR) [Huang et al. 2013a] is applied to upsample the surface points and then project the new samples that are close to the input data to the underlying surface using bilateral projection. This can effectively recover small geometry details; compare Figures 15(d) and (f) bottom. Furthermore, we can run bilateral normal smooth- 


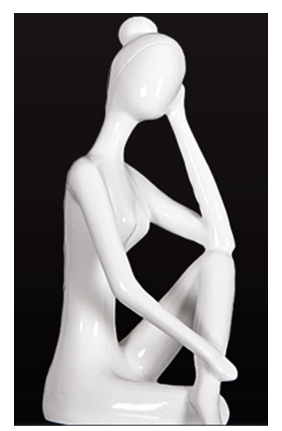

(a) Photo.

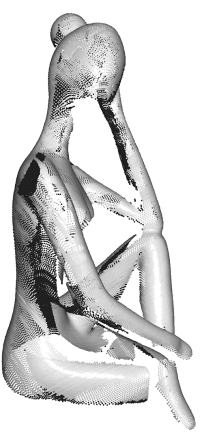

(b) Raw scan.

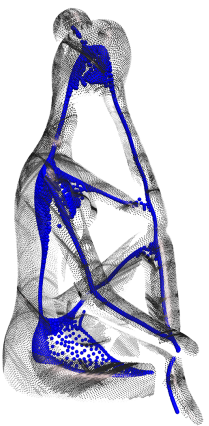

(c) Meso-skeleton

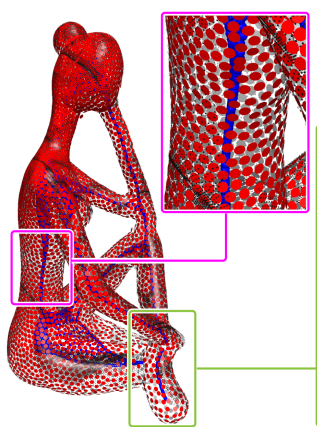

(d) Dpoints with $\sigma_{p}=5$
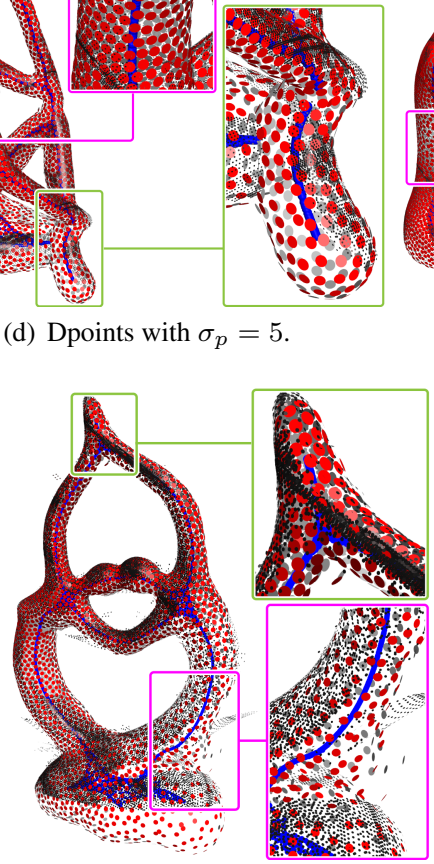

(i) Dpoints with $\sigma_{p}=5$.

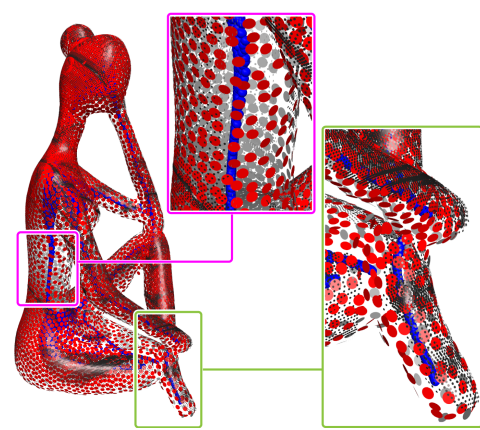

(e) Dpoints with $\sigma_{p}=3$.

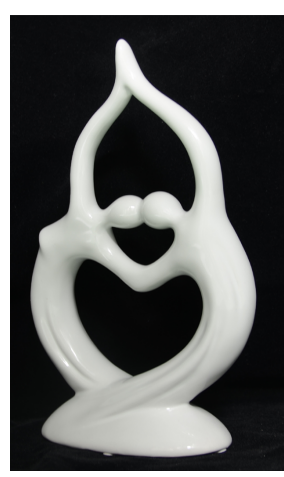

(f) Photo.

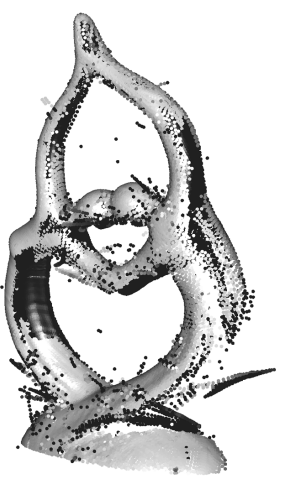

(g) Raw scan.

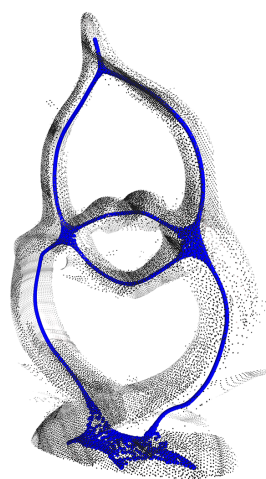

(h) Meso-skeleton.

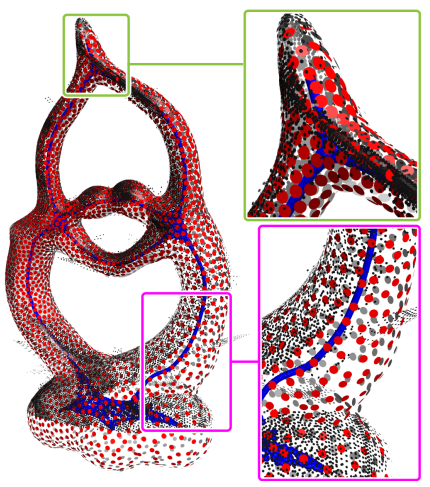

(j) Dpoints with $\sigma_{p}=8$.

Figure 10: The impact of the neighborhood size parameter $\sigma_{p}$. For shapes with complex topology, our approach generates proper mesoskeletons $(c)$ and $(h)$. In (d) under the default value $\sigma_{p}=5$, the partially scanned foot (green box) is noticeable thicker than the original model shown in (a). Lowering the value of $\sigma_{p}$ in $(e)$ makes the foot area closer to the original model, but the surface points on the body (pink box) are not as evenly distributed. In (i) due to noise in the raw scan ( $g$ ), some surface points (pink box) deviate away from the underlining surface under the default value. Increasing the value of $\sigma_{p}$ in $(j)$ makes the point set surface more robust against input noise, but does not represent the surface details well (green box).

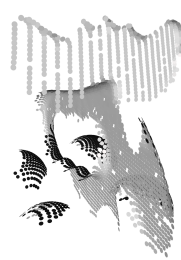

(a) 1 scan.

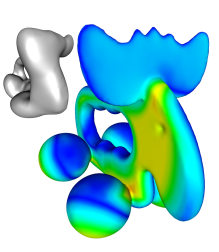

(b) Poisson of (a)

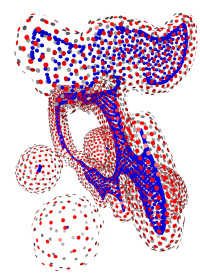

. (c) Dpoints of (a).

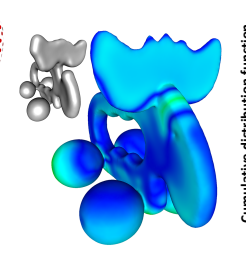

(d) Poisson of (c).

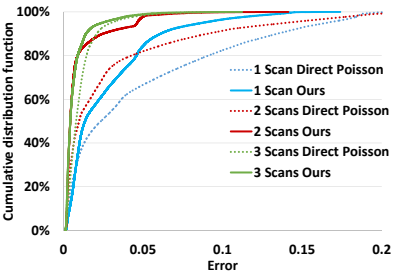

(e) Under clean scans.

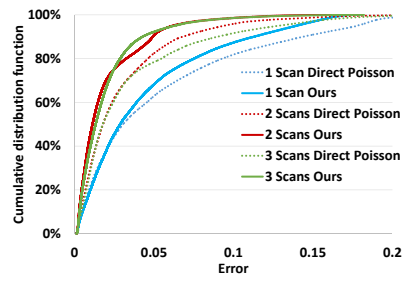

(f) Under noise-corrupted scans.

Figure 11: Quantitative evaluation on reconstruction accuracy using virtual scans of a ground truth synthetic model. When a single scan (a) is used, the direct Poisson reconstruction result (inset in (b)) does not resemble the model (shown in (b)). In comparison, the Poisson reconstructed model (inset in $(d)$ ) based on dpoints $(c)$ is visually much more accurate. The reconstruction errors, measured using the distances between vertices on the ground truth model and their closest points on the reconstructed surface, are visualized in $(b)$ and $(d)$. The error distributions under clean and noise-corrupted scans are plotted in $(e)$ and $(f)$, respectively.

ing to adjust the normals of surface points so that sharp features can be better preserved on the consolidated surface; compare Figures 15(d) and (f) top. In Figure 16 we show that our approach successfully reconstructs a shape with geometric features at different scales. While this leads to a disconnected meso-skeleton, as shown in blue in Figure 16(b), we can still reconstruct the small scale detail (pink box) as well as the large scale shape.
Shape priors. When the input scans contain open boundaries, e.g., the base of the models in Figures 9 and 10, our approach with the volume preserving shape prior (8) tries to close it through pushing surface points around the meso-skeleton and maintaining dpoints length $\left\|p_{i}-q_{i}\right\|$ to be similar to its neighbors. While such a strategy minimizes local volume variation, the reconstructed surface tends to have a "blobby" shape and may not accurately represent the real geometry of the missing surface, which is often flat and connects known surface through edges with $\mathcal{C}^{1}$ discontinuities. 

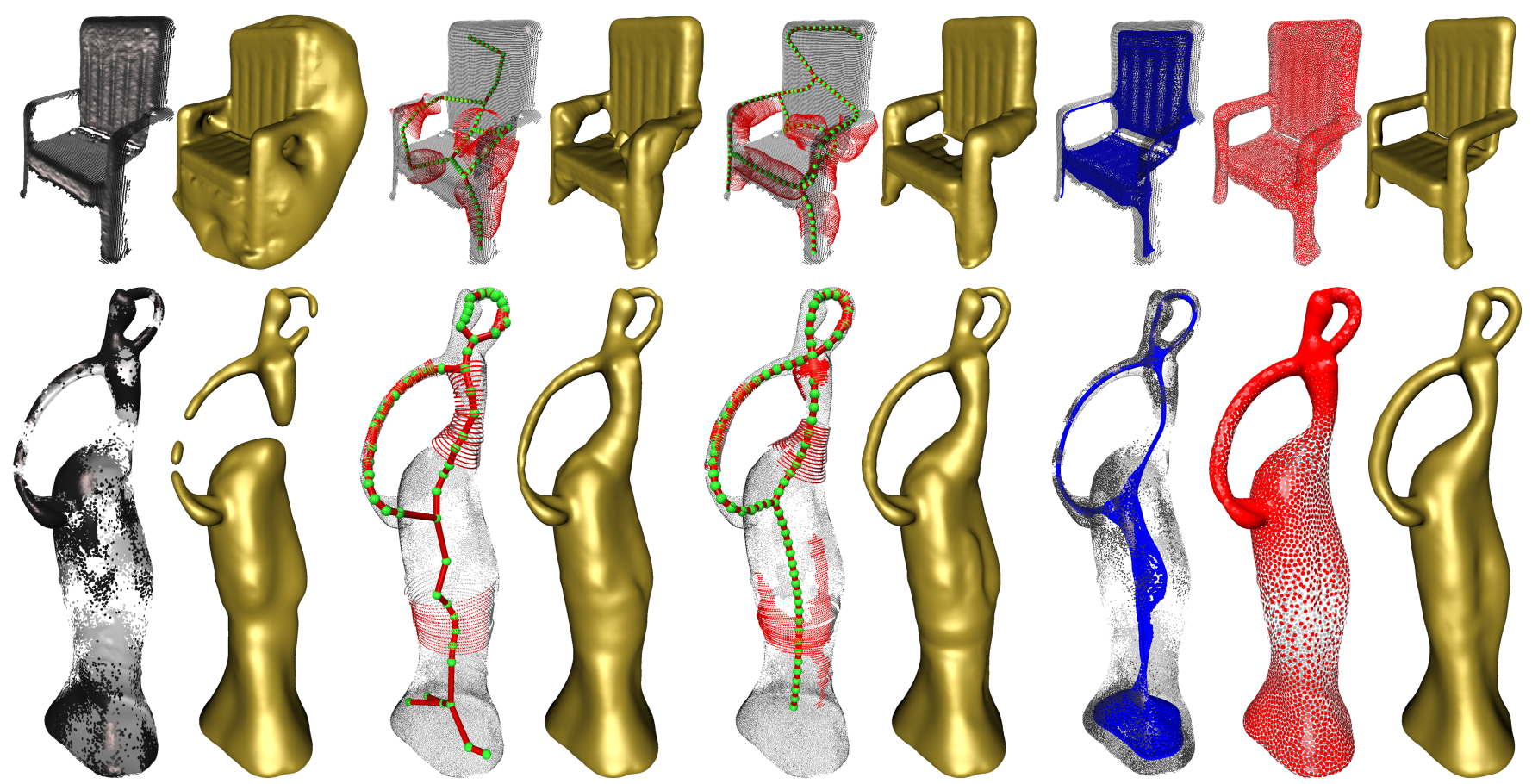

(a) Input + Poisson.

(b) ROSA + Poisson.

(c) $L_{1}$-medial + Poisson.

(d) Deep points + Poisson.

Figure 12: A comparison among the Poisson surface reconstructions [Kazhdan and Hoppe 2013] obtained using input points directly (a), ROSA skeleton [Tagliasacchi et al. 2009] (b), L L $_{1}$-medial skeleton [Huang et al. 2013b] (c), and our dpoints consolidation (d).
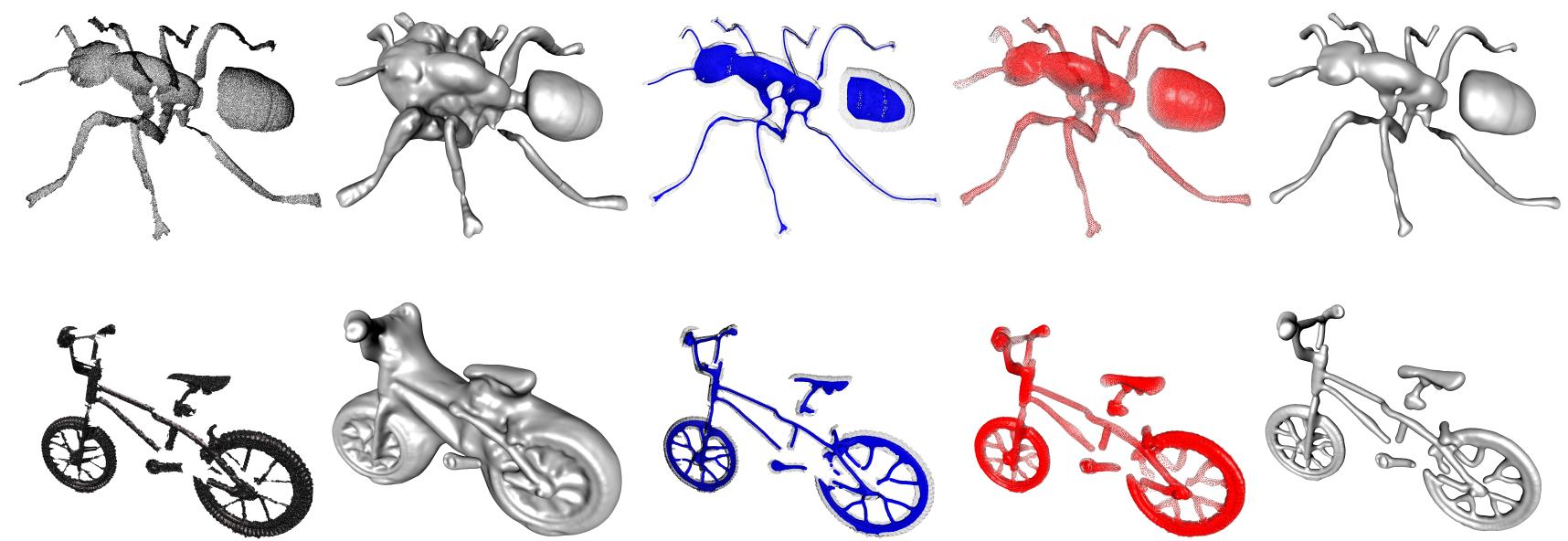

(a) Input scan.

(b) Poisson of (a).

(c) Skeletal points.

(d) Surface points.

(e) Poisson of (d).

Figure 13: Results on standard benchmark 3D scans (a), which are downloaded from the SHREC 2015 dataset [NIST 2015]. The direct Poisson reconstruction results (b) incorrectly fused multiple parts together. Using the consolidated dpoints ( $c \& d)$, the thin and adjacent structures are better preserved.

To address this problem, different shape priors shall be applied. For example, the minimal volume shape prior in Eq (9) described in Section 4.3 tries to close the surface so that the total volume of the shape is minimized. The dpoints representations generated using these two shape priors are compared in Figure 17, which demonstrates the difference between the consolidated point set surfaces, as well as the flexibility of our surface completion framework.
Limitations. In an effort to design a simple and elegant algorithm, our approach handles all operations through local point projections. No global smoothness is enforced for either points on the meso-skeleton or the consolidated surface points. Consequently, as shown in Figures 5 and 8, although our approach successfully connects the arms around the top of the model, the skeletal curve and the reconstructed point set surface do not appear to be smooth enough. Additional post-processing may therefore be needed.

Our method fails if the initial WLOP consolidation or the initial 

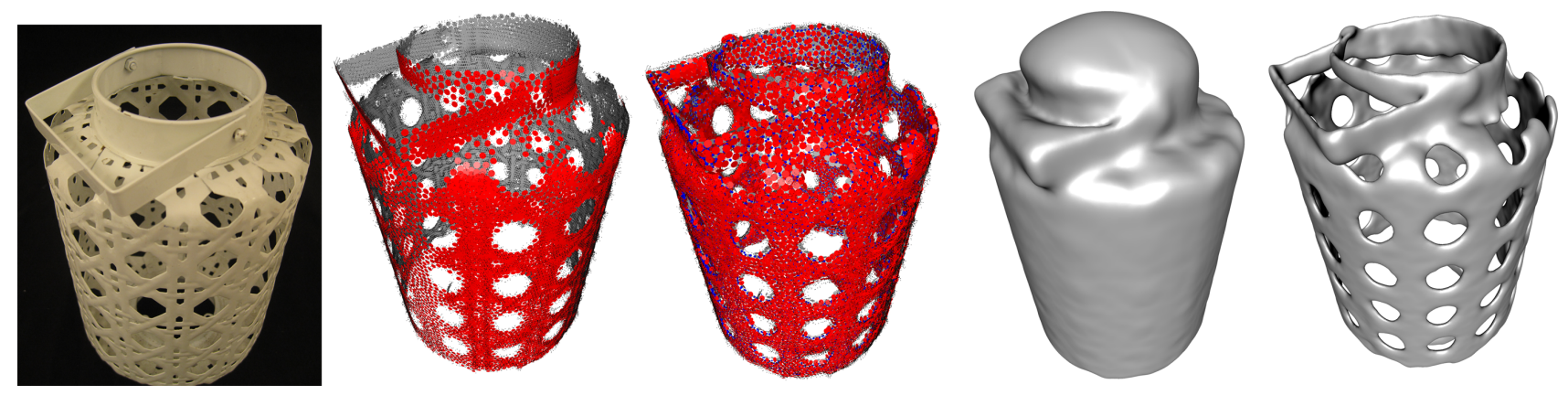

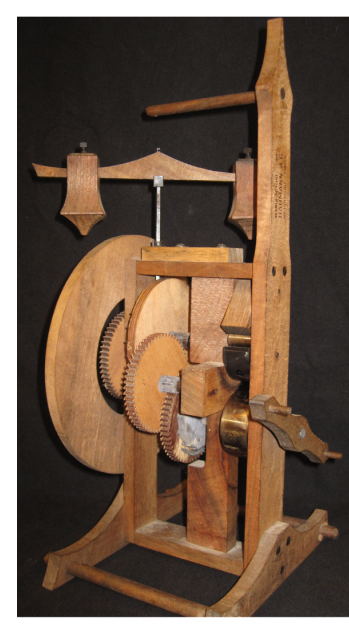

(a) Photo.

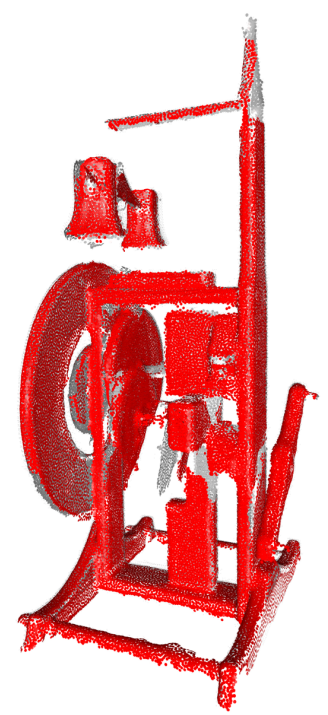

(b) Initial WLOP.

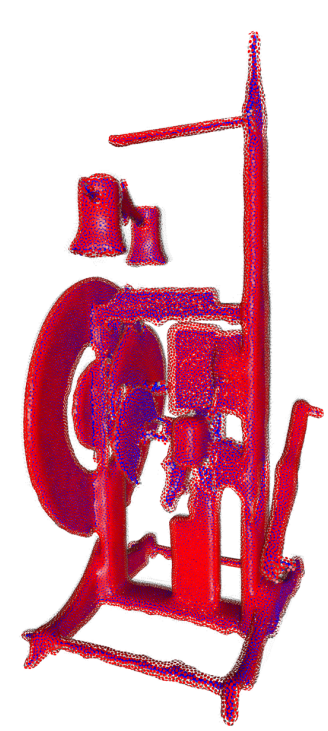

(c) Dpoints.

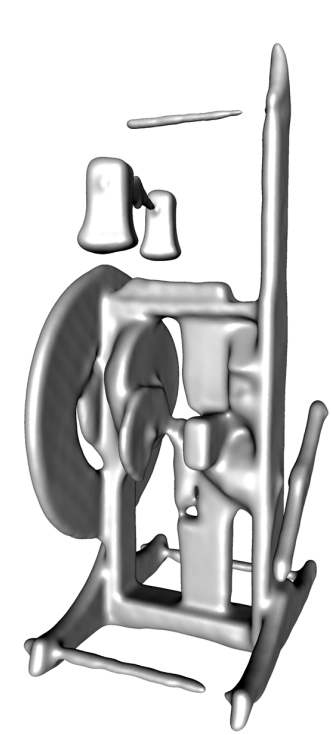

(d) Poisson of (b).

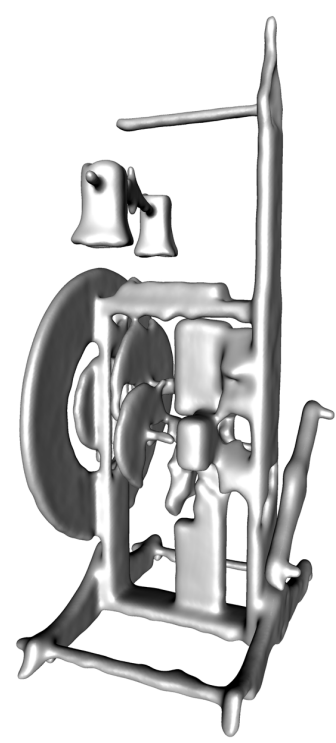

(e) Poisson of (c).

Figure 14: Handling objects (a) with complicated thin and non-tubular structures. Directly applying Poisson reconstruction over WLOP (b) failed to provide satisfying results $(d)$. Our reconstruction results (e) based on dpoints consolidation (c) better preserve the thin and non-tubular structures while maintaining the correct connectivity of different parts.
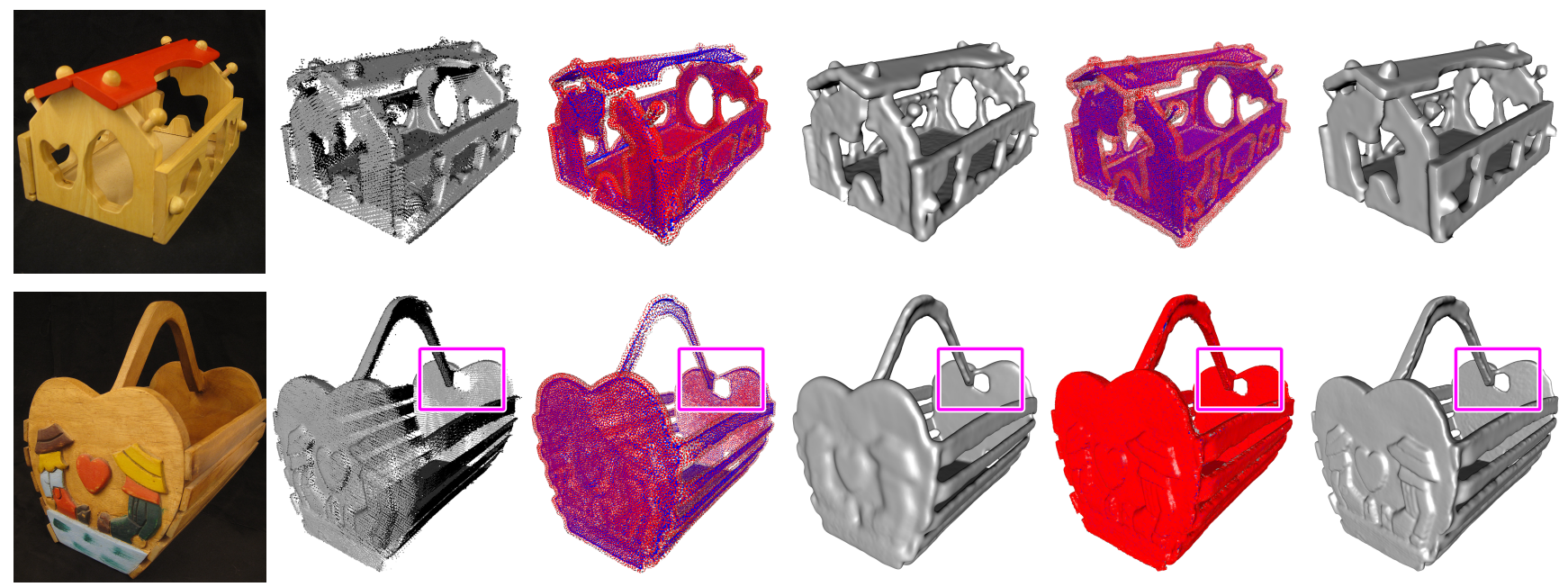

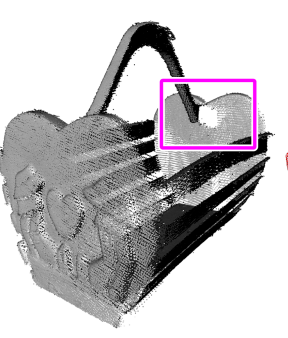

(b) Input scan.

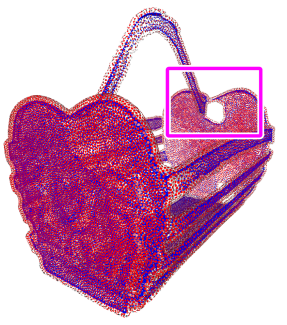

(c) Dpoints.

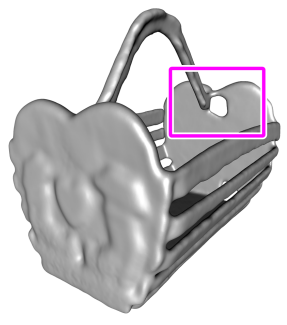

(d) Poisson of (c).

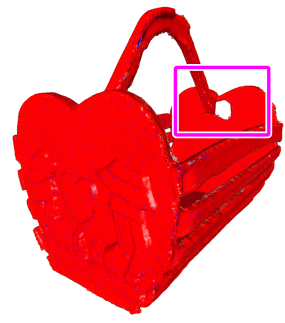

(e) EAR of (c).

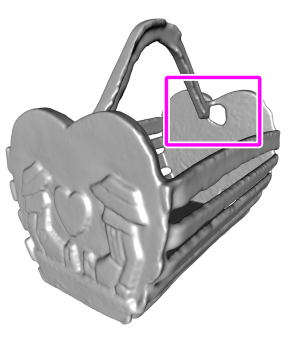

(f) Poisson of (e).

Figure 15: Post-processing for reconstructing fine geometry details and sharp features. While due to downsampling, the Poisson reconstruction results $(d)$ on dpoints $(c)$ cannot preserve fine details and sharp features as well as on the original shapes $(a, b)$, the post EAR [Huang et al. 2013a] step (e) effectively helps to recover them ( $f$ ) through inserting and projecting additional dpoints. 


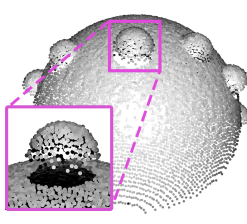

(a) Input scan.

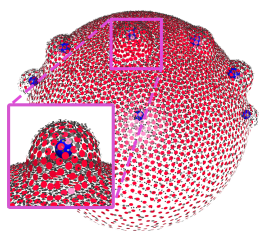

(b) Dpoints.

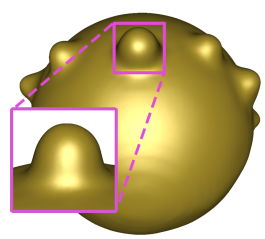

(c) Poisson of (c).
Figure 16: The dpoints representation preserves different scales of detail. While the meso-skeleton is disconnected, shown in blue in (b), the Poisson reconstruction in (c) captures both the large scale spherical shape as well as the small scale bumps.

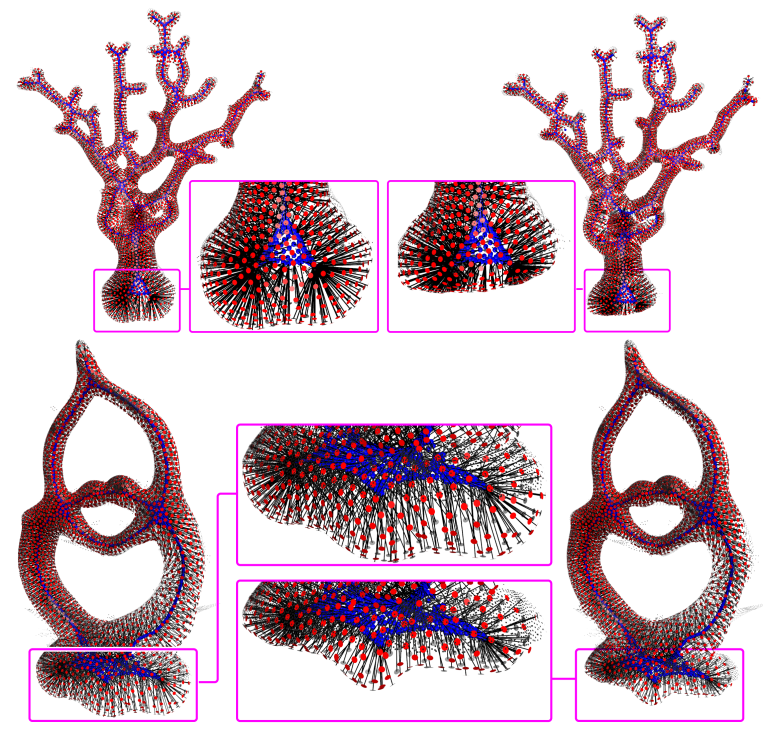

Figure 17: Dpoints representations under different shape priors. When there are $\mathcal{C}^{1}$ discontinuities between known and missing surfaces, using the volume preserving shape prior (left) results in "blobby" shapes. The minimal volume shape prior can be applied to alleviate this problem (right).

sinking stage fails. This may happen generally if either the data has too large missing parts, or the input contains very strong noise; see e.g., stress test results shown in supplementary materials. Our method always generates closed watertight point sets while preserving holes as shown in Figure 14 (top). On the other hand, we might mistakenly preserve holes due to large missing areas as boxed in Figure 15 (bottom). We believe that more accurately detecting and closing holes is a separate problem that would require stronger priors or user involvement.

\section{Conclusions and future work}

We present a novel representation for point sets, which facilitates the consolidation of noisy points and the completion of missing regions. The strength of the deep points representation comes from the fact that it is comprised of both local and non-local geometric information. For example, the dpoint orientations are used to adaptively determine the anisotropic neighborhoods when generating meso-skeletons and the dpoint lengths are used to control the local volume of the shape when consolidating surface points.

Deep points encode a consolidated point set surface and its mesoskeleton, and the mapping relations between the two in a highly concise representation. We believe that the non-local information that deep points carry is powerful for many applications. This is evident by the competence of skeletons and medial axis structures. In the future, we would like to explore the potential of using deep points representation for applications such as retrieval of pointbased geometries and deformation of point set surfaces.

\section{Acknowledgements}

We would like to thank the reviewers for their valuable feedback and SHREC 2015 for providing the datasets online. This work was supported in part by NSFC $(61522213,61232011,61379090)$, 973 Program (2014CB360503), 863 Program (2015AA016401), Guangdong Science and Technology Program (2015A030312015, 2014B050502009, 2014TX01X033), Shenzhen VisuCA Key Lab (CXB201104220029A), NSERC (293127) and BSF (2012376).

\section{References}

Adamson, A., And Alexa, M. 2006. Point-sampled cell complexes. ACM Trans. on Graphics (Proc. of SIGGRAPH) 25, 3, 671-680.

Alexa, M., Behr, J., Cohen-Or, D., Fleishman, S., LeVin, D., AND Silva, C. T. 2001. Point set surfaces. Proc. IEEE Int. Conf. on Visualization, 21-28.

Amenta, N., Bern, M., And Kamvysselis, M. 1998. A new voronoi-based surface reconstruction algorithm. In Proc. of SIGGRAPH, 415-421.

Au, O. K.-C., Tai, C.-L., Chu, H.-K., Cohen-Or, D., And LEE, T.-Y. 2008. Skeleton extraction by mesh contraction. ACM Trans. on Graphics (Proc. of SIGGRAPH) 27, 3, 44:1-44:10.

Avron, H., Sharf, A., Greif, C., and Cohen-Or, D. 2010. $\ell_{1}$-sparse reconstruction of sharp point set surfaces. ACM Trans. on Graphics 29, 5, 135:1-135:12.

Berger, M., Levine, J. A., Nonato, L. G., Taubin, G., And SILVA, C. T. 2013. A benchmark for surface reconstruction. ACM Trans. on Graphics (Proc. of SIGGRAPH) 32, 2, 20:120:17.

Berger, M., Tagliasacchi, A., Seversky, L. M., Alliez, P., Levine, J. A., Sharf, A., AND Silva, C. 2014. State of the art in surface reconstruction from point clouds. Eurographics STAR, 165-185.

BuCKsch, A., Lindenbergh, R., And Menenti, M. 2010. Skeltre: Robust skeleton extraction from imperfect point clouds. The Visual Computer 26, 10, 1283-1300.

Calderon, S., And BoubeKeur, T. 2014. Point morphology. ACM Trans. on Graphics (Proc. of SIGGRAPH) 33, 4, 45:145:13.

CaO, J., Tagliasacchi, A., Olson, M., Zhang, H., And Su, Z. 2010. Point cloud skeletons via laplacian based contraction. Proc. IEEE Int. Conf. on Shape Modeling \& Applications, 187197.

Carr, J. C., Beatson, R. K., Cherrie, J. B., Mitchell, T. J., Fright, W. R., McCallum, B. C., And Evans, T. R. 2001. Reconstruction and representation of 3D objects with radial basis functions. Proc. of SIGGRAPH, 67-76.

Cohen, L. D., And Cohen, I. 1991. Finite element methods for active contour models and balloons for $2 \mathrm{~d}$ and $3 \mathrm{~d}$ images. IEEE Trans. Pattern Analysis \& Machine Intelligence 15, 1131-1147. 
Davis, J., Marschner, S. R., Garr, M., And Levoy, M. 2002. Filling holes in complex surfaces using volumetric diffusion. In Proc. Int. Symp. on 3D Data Processing, Visualization and Transmission, 428-441.

Esteve, J., Brunet, P., And Vinacua, À. 2005. Computer Graphics Forum 24, 4, 791-807.

Guennebaud, G., And Gross, M. 2007. Algebraic point set surfaces. ACM Trans. on Graphics (Proc. of SIGGRAPH) 26, 3, 23.

Harary, G., TAl, A., AND Grinspun, E. 2014. Context-based coherent surface completion. ACM Trans. on Graphics 33, 1, 5:1-5:12.

Hoppe, H., DeRose, T., Duchamp, T., McDonald, J., And Stuetzle, W. 1992. Surface reconstruction from unorganized points. Proc. of SIGGRAPH, 71-78.

Huang, H., Li, D., Zhang, H., Ascher, U., AND COHEN-Or, D. 2009. Consolidation of unorganized point clouds for surface reconstruction. ACM Trans. on Graphics (Proc. of SIGGRAPH Asia) 28, 5, 176:1-176:7.

Huang, H., Wu, S., Gong, M., Cohen-Or, D., Ascher, U., AND ZHANG, H. 2013. Edge-aware point set resampling. ACM Trans. on Graphics 32, 1, 9:1-9:12.

Huang, H., Wu, S., Cohen-Or, D., Gong, M., Zhang, H., LI, G., AND CHEN, B. 2013. $l_{1}$-medial skeleton of point cloud. ACM Trans. on Graphics (Proc. of SIGGRAPH) 32, 4, 65:165:8.

KaZHDan, M., AND HopPe, H. 2013. Screened poisson surface reconstruction. ACM Trans. on Graphics 32, 1, 29:1-29:13.

Kazhdan, M., Bolitho, M., and Hoppe, H. 2006. Poisson surface reconstruction. Proc. Eurographics Symp. on Geometry Processing, 61-70.

LAnge, C., AND Polthier, K. 2005. Anisotropic smoothing of point sets. Computer Aided Geometric Design 22, 7, 680-692.

Li, G., LiU, L., Zheng, H., And Mitra, N. J. 2010. Analysis, reconstruction and manipulation using arterial snakes. $A C M$ Trans. on Graphics (Proc. of SIGGRAPH Asia) 29, 5, 152:1152:10.

Li, Y., Wu, X., Chrysathou, Y., Sharf, A., Cohen-Or, D., AND MitRA, N. J. 2011. Globfit: Consistently fitting primitives by discovering global relations. ACM Trans. on Graphics (Proc. of SIGGRAPH Asia) 30, 4, 52.

Lipman, Y., Cohen-Or, D., Levin, D., And Tal-Ezer, H. 2007. Parameterization-free projection for geometry reconstruction. ACM Trans. on Graphics (Proc. of SIGGRAPH) 26, 3, 22:1-22:6.

Livny, Y., Yan, F., Olson, M., Chen, B., Zhang, H., And EL-SANA, J. 2010. Automatic reconstruction of tree skeletal structures from point clouds. ACM Trans. on Graphics (Proc. of SIGGRAPH Asia) 29, 6, 151:1-151:8.

Miklos, B., Giesen, J., And Pauly, M. 2010. Discrete scale axis representations for 3D geometry. ACM Trans. on Graphics (Proc. of SIGGRAPH) 29, 4 (July), 101:1-101:10.

Natali, M., Biasotti, S., Patan, G., And Falcidieno, B. 2011. Graph-based representations of point clouds. Graphical Models 73, 151-164.
NIST. 2015. Range scans based 3D shape retrieval dataset. http://www.itl.nist.gov/iad/vug/sharp/contest/2015/Range/.

Öztireli, C., Guennebaud, G., And Gross, M. 2009. Feature preserving point set surfaces based on non-linear kernel regression. Computer Graphics Forum (Proc. of Eurographics) 28, 2, 493-501.

Pauly, M., Mitra, N. J., Wallner, J., Pottmann, H., And GuiBAS, L. J. 2008. Discovering structural regularity in 3D geometry. In ACM Trans. on Graphics (Proc. of SIGGRAPH), vol. $27,43: 1-43: 11$.

Preiner, R., Mattausch, O., Arikan, M., Pajarola, R., AND WIMmer, M. 2014. Continuous projection for fast $l_{1}$ reconstruction. ACM Trans. on Graphics (Proc. of SIGGRAPH) $33,4,47: 1-47: 13$.

Seversky, L. M., And Yin, L. 2012. A global parity measure for incomplete point cloud data. In Computer Graphics Forum (Proc. Pacific Conf. on Computer Graphics \& Applications), vol. 31, 2097-2106.

Sharf, A., Alexa, M., And Cohen-Or, D. 2004. Contextbased surface completion. ACM Trans. on Graphics (Proc. of SIGGRAPH) 23, 3, 878-887.

Sharf, A., Lewiner, T., Shamir, A., And Kobbelt, L. 2007. On-the-fly curve-skeleton computation for 3D shapes. Computer Graphics Forum 26, 323-328.

Sharf, A., Lewiner, T., Shklarski, G., Toledo, S., And COHEN-OR, D. 2007. Interactive topology-aware surface reconstruction. ACM Trans. on Graphics (Proc. of SIGGRAPH) 26, 3, 43:1-43:9.

Taglias acchi, A., Zhang, H., And Cohen-Or, D. 2009. Curve skeleton extraction from incomplete point cloud. $A C M$ Trans. on Graphics (Proc. of SIGGRAPH) 28, 3, 79:1-79:9.

Tagliasacchi, A., Olson, M., Zhang, H., Hamarneh, G., AND COHEN-OR, D. 2011. Vase: Volume-aware surface evolution for surface reconstruction from incomplete point clouds. In Computer Graphics Forum (Proc. Eurographics Symp. on Geometry Processing), vol. 30, 1563-1571.

Tagliasacchi, A., Alhashim, I., Olson, M., And Zhang, H. 2012. Mean curvature skeletons. Computer Graphics Forum (Proc. Eurographics Symp. on Geometry Processing) 31, 5, 1735-1744.

TURK, G., AND LEVOY, M. 1994. Zippered polygon meshes from range images. In Proc. of SIGGRAPH, 311-318.

Wu, S., Sun, W., Long, P., Huang, H., Cohen-Or, D., Gong, M., Deussen, O., And Chen, B. 2014. Qualitydriven poisson-guided autoscanning. ACM Trans. on Graphics (Proc. of SIGGRAPH Asia) 33, 6, 203:1-203:12.

Yin, K., Huang, H., Gong, M., Lischinski, D., COHEn-OR, D., Ascher, U., AND Chen, B. 2014. Morfit: Interactive surface reconstruction from incomplete point clouds with curvedriven topology and geometry control. ACM Trans. on Graphics (Proc. of SIGGRAPH Asia) 33, 6, 202:1-202:12.

Zheng, Q., Sharf, A., Wan, G., Li, Y., Mitra, N. J., Chen, B., AND COHEN-OR, D. 2010. Non-local scan consolidation for 3D urban scene. ACM Trans. on Graphics (Proc. of SIGGRAPH) 29, 94:1-94:10. 